\title{
Infinitely generated pseudocompact modules for finite groups and Weiss' Theorem
}

\author{
John William MacQuarrie ${ }^{1}$, Peter Symonds ${ }^{2}$, and Pavel Zalesskii ${ }^{3}$ \\ ${ }^{1}$ Universidade Federal de Minas Gerais, john@mat.ufmg.br \\ ${ }^{2}$ University of Manchester, peter.symonds@manchester.ac.uk \\ ${ }^{3}$ Universidade de Brasília, pz@mat.unb.br
}

February 11, 2020

\section{Introduction}

In 1988, A. Weiss [36] proved one of the most beautiful theorems in integral representation theory: it characterizes the finitely generated $\mathbb{Z}_{p}$-permutation lattices for a finite $p$-group $G$ in terms of information about the restriction to a normal subgroup $N$ and the action of $G / N$ on the $N$-invariants of the module (cf. also [37, §6], 28]). In 1993, Weiss generalized his own result to finite extensions of $\mathbb{Z}_{p}$, yielding the following theorem:

Theorem 1.1 ([38, [26, App. 1]). Let $R$ be a finite extension of $\mathbb{Z}_{p}$, let $G$ be a finite p-group and let $U$ be a finitely generated $R G$-lattice. Suppose there is a normal subgroup $N$ of $G$ such that

- the module $U$ restricted to $N$ is a free $R N$-module,

- the submodule of fixed points $U^{N}$ is a permutation $R G$-module.

Then $U$ itself is a permutation $R G$-module.

The importance of this theorem is highlighted by its applications. Weiss originally proved it in order to show that every finite $p$-subgroup of augmentation- 1 units in $R G$ is conjugate to a subgroup of $G$ 36. Puig used it to show that if a block of the group algebra $R F$ of a finite group $F$ is stably Morita equivalent to a nilpotent block then it is nilpotent [26, Thm 8.2]. No alternative proofs of these results are known. We generalize Theorem 1.1 in two ways. First, we allow the coefficient ring $R$ to be any complete discrete valuation ring in mixed characteristic (that is, with residue field of prime characteristic $p$ and with field of fractions of characteristic 0 ). The second generalization is more important: we prove the result for all pseudocompact $R G$-lattices, possibly infinitely generated. What is important here is that the rank of the lattice is allowed to be infinite. Pseudocompact is just the correct generalization of profinite to the case when $R$ is not profinite; we work with such modules because they are better behaved than abstract ones in the infinitely generated case.

The notion of permutation profinite modules was introduced by Mel'nikov in [25], where he studied their basic properties. As he pointed out, profinite permutation modules are important for the combinatorial theory of profinite groups and especially of pro- $p$ groups. A different approach, which complements the approach of Mel'nikov in the study of the basic properties of profinite permutation modules, was introduced by the second author [33, where these modules were used to develop the cohomology theory of profinite groups along the lines followed for discrete groups, as in the book of Brown 8 . We note also that permutation profinite modules appear naturally in Galois Theory [2, Thm 1.3].

Before stating our main theorem for a finite $p$-group $G$ we define a pseudocompact permutation $R G$ lattice $U$ to be a pseudocompact module that is free over $R$ and having a pointed compact $G$-invariant basis (several alternative definitions are given in detail and compared in Section 2.3). 
Theorem 1.2. Let $R$ be a complete discrete valuation ring in mixed characteristic with residue field of characteristic $p$, let $G$ be a finite p-group and let $U$ be a pseudocompact $R G$-lattice. Suppose there is a normal subgroup $N$ of $G$ such that

- the module $U$ restricted to $N$ is a free $R N$-module,

- the submodule of fixed points $U^{N}$ is a permutation $R G$-module.

Then $U$ itself is a permutation $R G$-module.

This theorem plays a central role in the proof of the pro- $p$ version 39] of the theorem of Karras, Pietrowski, Solitar, Cohen and Scott [19, 12, 31, which states that a virtually free group acts on a tree with finite vertex stabilizers. Indeed, in the pro- $p$ case Theorem 1.2 replaces Stallings' theory of ends, crucial in the proof of the original result. Recently, the greater generality of coefficient ring has yielded applications of Theorem 1.2 in the calculation of Picard groups of blocks of finite groups [6, 16, 21.

One might expect that the generalization to infinite rank lattices could be proved using a simple limit argument, but this does not seem to be the case. Instead, we need to recast the proof in a way that does not depend on expressing a module as a sum of indecomposable modules. In order to do this we look closely at the properties of various classes of infinitely generated modules; these results should be of independent interest.

We make extensive use of relative homological algebra, and in particular of the concepts of covers and precovers of a module by a module with desirable properties. We prove the existence of a large class of covers of pseudocompact modules (Theorem 6.2). We give an explicit description of the permutation cover of a pseudocompact module for a finite group when $R$ is a complete discrete valuation ring (Theorem 6.13). We also generalize a related theorem of Cliff and Weiss (Theorem 7.2).

\section{Acknowledgements}

The authors thank the anonymous referee, whose thorough reading of the article has significantly improved the exposition in several places. The first author was partially supported by a CNPq Universal Grant, a UFMG Recém Contratados grant and a FAPEMIG PPM Grant. The second author was partially supported by an International Academic Fellowship from the Leverhulme Trust. The third author wishes to acknowledge the financial support of CNPq and FAPDF.

\section{Definitions, terminology and background}

\section{$2.1 \quad$ Algebras and modules}

Our coefficient ring $R$ will always be a commutative pseudocompact ring. In later sections we will require further structure on $R$, the main coefficient rings of interest to us being complete discrete valuation rings.

Let $\Lambda$ be a pseudocompact $R$-algebra (we follow the treatments in 9 and [18). Examples of particular interest are the completed group algebra $R[G]$ ] of a profinite group $G$, or later the group algebra $R G$ of a finite group $G$. We consider the following categories of modules for $\Lambda$ :

- $\Lambda-\mathrm{Mod}^{\mathcal{C}}$ : the category whose objects are pseudocompact left $\Lambda$-modules [9, $\left.\S 1\right]$. Objects of this category are complete, Hausdorff topological $\Lambda$-modules $U$ having a basis of open neighbourhoods of 0 consisting of submodules $V$ for which $U / V$ has finite length. In other words, the category of inverse limits of left $\Lambda$-modules of finite length over $R$.

- $\Lambda$-Mod ${ }^{\mathcal{D}}$ : the category whose objects are those topological $\Lambda$-modules having the discrete topology. Such modules are precisely the direct limits of left $\Lambda$-modules of finite length over $R$. We will call such modules discrete (they are called "locally finite" in [18]).

- $\Lambda$-Mod ${ }^{\text {abs }}$ : the category of abstract left $\Lambda$-modules 
Morphisms in $\Lambda$ - $\operatorname{Mod}^{\mathcal{C}}$ and $\Lambda$-Mod ${ }^{\mathcal{D}}$ are continuous $\Lambda$-module homomorphisms while morphisms in $\Lambda$-Mod ${ }^{\text {abs }}$ are arbitrary $\Lambda$-module homomorphisms. The corresponding categories of right modules are denoted $\operatorname{Mod}^{\mathcal{C}}-\Lambda, \operatorname{Mod}^{\mathcal{D}}-\Lambda$ and $\operatorname{Mod}^{\text {abs }}-\Lambda$. We include the decorations $\mathcal{C}, \mathcal{D}$, abs inconsistently, omitting them when the category is clear from the context. We occasionally write $\mathrm{Hom}^{\text {cts }}$ or Hom ${ }^{\text {abs }}$ to make explicit whether we consider continuous or arbitrary homomorphisms. A $\Lambda$-lattice is a $\Lambda$-module in $\Lambda-\operatorname{Mod}^{\mathcal{C}}, \Lambda-\operatorname{Mod}^{\text {abs }}$ or in $\Lambda-\operatorname{Mod}^{\mathcal{D}}$ that is projective as an $R$-module in the corresponding category. Note that the way sums and products appear is not quite as one might expect from the abstract case - for example, free pseudocompact $\Lambda$-modules are direct products of copies of $\Lambda$ (cf. $[9, \S 1]$ ).

Following [9], denote by $E_{R}$ the dualizer of $R$ : that is, $E_{R}$ is the injective hull in $R$-Mod ${ }^{\mathcal{D}}$ of the module

$$
\bigoplus_{\mathfrak{m}} R / \mathfrak{m}
$$

where $\mathfrak{m}$ runs through the maximal ideals of $R$. The functor sending a module $M$ (in $\Lambda$-Mod ${ }^{\mathcal{C}}$ or $\operatorname{Mod}^{\mathcal{D}}-\Lambda$ ) to the module of continuous $R$-module homomorphisms from $M$ to $E_{R}$ induces a Pontryagin duality between the categories $\Lambda-\operatorname{Mod}^{\mathcal{C}}$ and $\operatorname{Mod}^{\mathcal{D}}-\Lambda$ [9, Prop.2.3] so that any result in one category corresponds to a dual result in the other. We will at times use this observation without comment. Given a module $M$ in either the pseudocompact or the discrete category, we denote its Pontryagin dual by $M^{*}$.

Denote by $\Lambda$-Proj (resp. $\Lambda$-Inj) the full subcategories of (a given category of) left $\Lambda$-modules having as objects the projective modules (resp. injective modules). The category $\Lambda$-Mod ${ }^{\mathcal{C}}$ has projective covers and the category $\Lambda-\mathrm{Mod}^{\mathcal{D}}$ has injective hulls [18, Ch.II, Thm 2]. An arbitrary product of projective modules in $\Lambda-\operatorname{Mod}^{\mathcal{C}}$ is projective and an arbitrary sum of injective modules in $\Lambda$-Mod $\mathcal{D}$ is injective (the first statement follows from [9, Cor. 3.3] and the second is its dual).

Given a module $M$ in $\Lambda$-Mod ${ }^{\mathcal{C}}$, denote by $\operatorname{Add}^{\mathcal{C}}(M)$ the full subcategory of $\Lambda$-Mod $\mathcal{C}^{\mathcal{C}}$ whose objects are summands of products of $M$. Denote by $\operatorname{add}^{\mathcal{C}}(M)$ the full subcategory of $\operatorname{Add}^{\mathcal{C}}(M)$ consisting of summands of finite products of $M$. Dually, given a module $M$ in $\Lambda-\operatorname{Mod}^{\mathcal{D}}$ we have the categories $\operatorname{Add}^{\mathcal{D}}(M)$ of summands of direct sums of $M$ and $\operatorname{add}^{\mathcal{D}}(M)$ whose objects are summands of finite sums of $M$. If $M \in \Lambda-\operatorname{Mod}^{\mathcal{C}}$, the category dual to $\operatorname{Add}^{\mathcal{C}}(M)$ is $\operatorname{Add}^{\mathcal{D}}\left(M^{*}\right)$.

Nakayama's Lemma holds in the category $R$-Mod ${ }^{\mathcal{C}}$ : if $N$ is a closed submodule of the pseudocompact $R$-module $M$ such that $N+\operatorname{Rad}(R) M=M$, where $\operatorname{Rad}(R)$ is the intersection of the maximal ideals of $R$, then $M=N$ [9, Lem. 1.4]. There is a dual version for $R-\operatorname{Mod}^{\mathcal{D}}$.

It is convenient to be able to describe a $\Lambda$-lattice in terms of $\Lambda$-lattices of finite rank over $R$. We prove only a special case that we will require later.

Lemma 2.1. Let $G$ be a finite group, $R$ a complete discrete valuation ring and $U$ a pseudocompact $R G$ lattice. Then $U$ can be expressed as the inverse limit of an inverse system of $R G$-lattices of finite rank with surjective homomorphisms.

Proof. As an $R$-lattice, we can write $U=\prod_{i \in I} R$. For each cofinite subset $J$ of $I$, let $U_{J}=\prod_{i \in J} R$ and $V_{J}=\bigcap_{g \in G} g U_{J}$. Then $V_{J}$ is an $R G$-submodule of $U$. The obvious homomorphism

$$
U / V_{J} \rightarrow \bigoplus_{g \in G} U / g U_{J}
$$

is injective, so $U / V_{J}$ is isomorphic as an $R$-module to a submodule of an $R$-lattice of finite rank, hence it is an $R G$-lattice of finite rank itself, since $R$ is a discrete valuation domain.

Because $V_{J} \subseteq U_{J}$ and

$$
U=\lim _{J} U / U_{J}
$$

we have

$$
U=\lim _{J} U / V_{J}
$$




\subsection{Tensor products, homomorphisms, Ext functors}

The references in this section are sometimes incomplete, insofar as the proofs given are for profinite, rather than pseudocompact modules. What we mean is that the proof given in the reference also works for pseudocompact modules.

Let $\Lambda, \Gamma$ be pseudocompact $R$-algebras, $M$ a pseudocompact $\Lambda$ - $\Gamma$-bimodule (that is, $M$ is a bimodule that is pseudocompact both as a left $\Lambda$-module and as a right $\Gamma$-module) and $N$ a pseudocompact left $\Gamma$-module. The completed tensor product defined in $[9, \S 2]$ is a pseudocompact $R$-module $M \widehat{\otimes}_{\Gamma} N$ together with a continuous bilinear map $M \times N \rightarrow M \widehat{\otimes}_{\Gamma} N$, written $(m, n) \mapsto m \widehat{\otimes} n$ satisfying the condition that $m g \widehat{\otimes} n=m \widehat{\otimes} g n$ for all $m \in M, n \in N, g \in \Gamma$ and universal with respect to this condition. The completed tensor product inherits naturally the structure of a left $\Lambda$-module with multiplication given on pure tensors by $x(m \widehat{\otimes} n):=x m \widehat{\otimes} n$. If $M=\varliminf_{\varlimsup} M_{i}, N=\lim _{j} N_{i}$ are expressions of $M, N$ as inverse limits of $\Gamma$-modules of finite $R$-length, then

$$
M \widehat{\otimes}_{\Gamma} N=\lim _{i, j} M_{i} \otimes N_{j}
$$

27. Lem. 5.5.1]. The completed tensor product commutes with inverse limits in both variables 27, Lem. 5.5.2]. It is not, in general, isomorphic to the abstract tensor product, but we do have the following result.

Proposition 2.2. If either $M$ or $N$ is finitely presented as a $\Gamma$-module, or if both $M$ and $N$ are finitely generated as $\Gamma$-modules, then the natural map $M \otimes_{\Gamma} N \rightarrow M \widehat{\otimes}_{\Gamma} N$ is an isomorphism.

Proof. The result for finitely presented modules is [14, Lem. 1.1]. When both modules are finitely generated, one need only observe that the left-most vertical map in the proof of [14, Lem. 1.1 (iii)] is still surjective, so the same proof works.

Note that the assertion in [9, Lem. 2.1 (ii)] that the tensor products $\widehat{\otimes}$ and $\otimes$ coincide when only one of the modules involved is finitely generated is false in general [14, Corol. 2.4].

If $M$ is a topological $\Lambda$ - $\Gamma$-bimodule and $N$ is a topological left $\Lambda$-module, $\operatorname{Hom}_{\Lambda}(M, N)$ denotes the left $\Gamma$-module of continuous $\Lambda$-module homomorphisms from $M$ to $N$ and is given the compact-open topology. If $M, N$ are pseudocompact, then $\operatorname{Hom}_{\Lambda}(M, N)=\lim _{i} \operatorname{Hom}_{\Lambda}\left(M, N_{i}\right)$, where $N=\lim _{i} N_{i}$ and each $N_{i}$ has finite length over $R$. The compact-open topology coincides with the topology obtained by declaring each $\operatorname{Hom}_{\Lambda}\left(M, N_{i}\right)$ to be discrete and giving $\operatorname{Hom}_{\Lambda}(M, N)$ the inverse limit topology. In particular, $\operatorname{Hom}_{\Lambda}(M, N)$ commutes with products in the second variable. We also have that $\operatorname{Hom}_{\Lambda}(M, N)$ commutes with finite products in the first variable. If $M$ is finitely generated as a $\Lambda$-module and $N$ is either pseudocompact or discrete, then every abstract homomorphism from $M$ to $N$ is continuous. If $M$ is finitely generated and $N$ is pseudocompact then $\operatorname{Hom}_{\Lambda}(M, N)$ is pseudocompact as an $R$-module. Furthermore:

Lemma 2.3. If $M$ is a finitely generated pseudocompact $\Lambda$-module, then $E=\operatorname{End}_{\Lambda}(M)$ is a pseudocompact $R$-algebra.

Proof. Write $\Lambda=\lim \Lambda / I_{i}$ with each $\Lambda / I_{i}$ of finite length over $R$. Then $M / \mathrm{Cl}\left(I_{i} M\right)$ has finite length over $R$ (where $\mathrm{Cl}$ denotes topological closure) and hence so does $\operatorname{End}_{\Lambda}\left(M / \mathrm{Cl}\left(I_{i} M\right)\right)$. Let $J_{i}$ denote the two sided ideal $\left\{\rho \in E \mid \rho(M) \leqslant \mathrm{Cl}\left(I_{i} M\right)\right\}$ of $E$. The map $E / J_{i} \rightarrow \operatorname{End}_{\Lambda}\left(M / \mathrm{Cl}\left(I_{i} M\right)\right)$ is injective, so each $E / J_{i}$ has finite length over $R$. But $E=\lim _{\longleftarrow} E / J_{i}$ and hence $E$ is pseudocompact.

Let $X$ be a pseudocompact $\Lambda$ - $\Gamma$-bimodule that is finitely generated as a left $\Lambda$-module. The functor

$$
X \widehat{\otimes}_{\Gamma}-: \Gamma-\operatorname{Mod}^{\mathcal{C}} \rightarrow \Lambda-\operatorname{Mod}^{\mathcal{C}}
$$

is left adjoint to the functor

$$
\operatorname{Hom}_{\Lambda}(X,-): \Lambda-\operatorname{Mod}^{\mathcal{C}} \rightarrow \Gamma-\operatorname{Mod}^{\mathcal{C}}:
$$


given a pseudocompact left $\Gamma$-module $A$ and a pseudocompact left $\Lambda$-module $C=\lim C_{i}$ with each $C_{i}$ of finite length, we have

$$
\begin{aligned}
\operatorname{Hom}_{\Gamma}\left(A, \operatorname{Hom}_{\Lambda}\left(X, \varliminf_{\longleftarrow} C_{i}\right)\right) & \cong \operatorname{Hom}_{\Gamma}\left(A, \lim _{\leftarrow} \operatorname{Hom}_{\Lambda}\left(X, C_{i}\right)\right) \\
& \cong \lim _{\leftarrow} \operatorname{Hom}_{\Gamma}\left(A, \operatorname{Hom}_{\Lambda}\left(X, C_{i}\right)\right) \\
& \cong \lim _{\leftarrow} \operatorname{Hom}_{\Lambda}\left(X \widehat{\otimes}_{\Gamma} A, C_{i}\right) \\
& \cong \operatorname{Hom}_{\Lambda}\left(X \widehat{\otimes}_{\Gamma} A, \lim _{\longleftarrow} C_{i}\right),
\end{aligned}
$$

where the second to last isomorphism is [9, Lem. 2.4] (though note that the side conventions there are different). If $\Psi, \Phi$ are other pseudocompact algebras, $A$ is a pseudocompact $\Gamma$ - $\Phi$-module and $C$ is a pseudocompact $\Lambda$ - $\Psi$-bimodule, then the above isomorphisms are isomorphisms of $\Phi$ - $\Psi$-bimodules [5] Thm 4.2].

If $H$ is a closed subgroup of the profinite group $G$ and $V$ is a pseudocompact $R[[H]]$-module, the induced $R[[G]]$-module $V \uparrow^{G}$ is defined to be

$$
V \uparrow G:=R\left[[G] \widehat{\otimes}_{R[[H]]} V,\right.
$$

where $R[[G]]$ is treated as an $R[[G]]-R[[H]]$-bimodule in the obvious way. Given a pseudocompact $R[[G]]$ module $U$, the restriction $U \downarrow_{H}$ (that is, $U$ treated as an $R[[H]]$-module) can be expressed as $U \downarrow_{H}=$ $\operatorname{Hom}_{R \llbracket G \rrbracket}(R[[G]], U)$, where again $R[[G]]$ is being treated as an $R[[G]]-R[[H]]$-bimodule in the obvious way. It follows from the adjunction above that induction is left adjoint to restriction. When $H$ is open in $G$, induction is also right adjoint to restriction. To see this, we need only note that, since $H$ is open, restriction is anyway left adjoint to the coinduction functor $\operatorname{Hom}_{R \llbracket H \rrbracket}(R[G],-)$. But since $R \llbracket G \rrbracket$ is finitely presented as an $R[[H]]$-module, every functor involved coincides with its abstract counterpart. We may thus apply [35, Lem. 6.3.4]. Let $H, L$ be closed subgroups of the profinite group $G$ with either $H$ or $L$ open. The Mackey decomposition formula applies. That is, given a pseudocompact $R[[H]]$-module $V$, we have

$$
V \uparrow^{G} \downarrow_{L} \cong \bigoplus_{g \in L \backslash G / H}{ }^{g} V \downarrow_{g H \cap L} \uparrow^{L}
$$

where $g$ runs through a (finite) set of double coset representatives of $L \backslash G / H$ and ${ }^{g} V$ is the $\left.R\left[{ }^{g} H\right]\right]$-module $V$ with action $g h g^{-1} \cdot v=h v$.

Given pseudocompact $\Lambda$-modules $M, N$, denote by $\operatorname{Ext}_{\Lambda}^{i}(M, N)$ the $i$ th right derived functor of $\operatorname{Hom}_{\Lambda}(-, N)$ applied to $M$. It can be calculated via a projective resolution for $M$ in the usual way. Since $\mathrm{Hom}_{\Lambda}$ commutes with finite products in the first variable and arbitrary products in the second, it follows that $\operatorname{Ext}_{\Lambda}^{i}(M, N)$ also commutes with finite products in $M$ and arbitrary products in $N$. However, it does not commute with arbitrary inverse limits in the second variable. Since $\Lambda$-Mod $\operatorname{Mod}^{\mathcal{C}}$ is abelian and has enough projectives, the functor $\operatorname{Ext}_{\Lambda}^{1}$ classifies extensions as one would hope [35, Vis. 3.4.6].

Dually, in $\Lambda$-Mod ${ }^{\mathcal{D}}$, denote by $\operatorname{Ext}_{\Lambda}^{i}(M, N)$ the $i$ th right derived functor of $\operatorname{Hom}_{\Lambda}(M,-)$ applied to $N$. By Pontryagin duality,

$$
\operatorname{Ext}_{\Lambda-\operatorname{Mod}^{\mathcal{C}}}^{i}(M, N) \cong \operatorname{Ext}_{\operatorname{Mod}^{\mathcal{D}}-\Lambda}^{i}\left(N^{*}, M^{*}\right) .
$$

In what follows, when we say that $\Lambda$ is finitely generated over $R$, we mean finitely generated as an $R$-module. Examples of particular interest are $\Lambda=R G$ for a finite group $G$.

Lemma 2.4. Suppose $\Lambda$ is finitely generated over $R$. If $M, N$ are pseudocompact $\Lambda$-modules with $M$ finitely generated, and if either $R$ is noetherian or $M$ is a lattice, then

$$
\operatorname{Ext}_{\Lambda-\operatorname{Mod}^{\mathcal{C}}}^{i}(M, N) \cong \operatorname{Ext}_{\Lambda-\operatorname{Mod}^{\text {abs }}}^{i}(M, N) .
$$

Proof. Our conditions guarantee that there exists a projective resolution of $M$ in $\Lambda$-Mod ${ }^{\mathcal{C}}$ with each module finitely generated. This is also a projective resolution of $M$ by projective modules in $\Lambda$-Mod ${ }^{\text {abs }}$. But $\operatorname{Hom}_{\Lambda-\operatorname{Mod}^{c}}(P, N)=\operatorname{Hom}_{\Lambda-\operatorname{Mod}^{a b s}}(P, N)$ whenever $P$ is finitely generated (that is, every abstract homomorphism $P \rightarrow N$ is continuous), and so the groups obtained are the same. 
Lemma 2.5. Suppose that $R$ is noetherian and $\Lambda$ is finitely generated over $R$. For $M, N \in \Lambda$-Mod ${ }^{\mathcal{D}}$ we have

$$
\operatorname{Ext}_{\Lambda-\operatorname{Mod}^{\mathcal{D}}}^{i}(M, N) \cong \operatorname{Ext}_{\Lambda-\operatorname{Mod}^{\text {abs }}}^{i}(M, N) .
$$

Proof. By taking the Pontryagin dual of a free resolution of the pseudocompact module $N^{*}$, we obtain an injective resolution of $N$ by modules that are sums of modules of the form $\Lambda^{*}=\operatorname{Hom}_{R-\operatorname{Mod}}^{\text {cts }}\left(\Lambda, E_{R}\right)$. Since $\Lambda$ is finitely generated over $R$, we have $\operatorname{Hom}_{R-\operatorname{Mod}}^{\mathrm{cts}}\left(\Lambda, E_{R}\right)=\operatorname{Hom}_{R \text {-Mod }}^{\text {abs }}\left(\Lambda, E_{R}\right)$. The injective hull in $R$-Mod ${ }^{\text {abs }}$ of the module $R / \mathfrak{m}$ ( $\mathfrak{m}$ maximal ideal of $R$ ) is locally finite, since every element is annihilated by some power of $\mathfrak{m}$ by [24, Thm 18.4] and $R / \mathfrak{m}^{n}$ has finite length over $R$ for any $n \in \mathbb{N}$ because $R$ is noetherian. Hence the injective hulls of $R / \mathfrak{m}$ in the abstract and the discrete categories coincide. Furthermore, $R$ being noetherian implies that a direct sum of injective modules in the abstract category is injective [24, Thm 18.5]. We conclude that $E_{R}$ is injective in $R$-Mod ${ }^{\text {abs }}$.

Now, the functor $\operatorname{Hom}_{R \text {-Mod }}^{\text {abs }}(\Lambda,-): R$-Mod ${ }^{\text {abs }} \rightarrow \Lambda-\operatorname{Mod}^{\text {abs }}$ is a right adjoint by the theory of abstract modules, and hence by [35, Prop. 2.3.10], $\Lambda^{*}$ is injective in $\Lambda$-Mod ${ }^{\text {abs }}$.

Putting all this together, the above injective resolution of $N$ in $\Lambda-\operatorname{Mod}^{\mathcal{D}}$ is also an injective resolution of $N$ in $\Lambda-\operatorname{Mod}^{\text {abs }}$. Furthermore, the hom groups obtained by applying $\operatorname{Hom}_{\Lambda-\operatorname{Mod}^{\mathcal{D}}}(M,-)$ and $\operatorname{Hom}_{\Lambda-M o d}$ abs $(M,-)$ to this injective resolution are the same and so the Ext groups are the same.

As usual, when $G$ is a finite group we denote by $H^{n}(G,-)$ the $n$th right derived functor of the fixed point functor $(-)^{G}$. We require only the following basic version of the Eckmann-Shapiro Lemma, which can be proved in the usual way given that the induction and coinduction functors coincide for finite groups: When $H$ is a subgroup of $G$ and $V$ is a pseudocompact $R H$-module, then $H^{n}(H, V) \cong H^{n}\left(G, V \uparrow^{G}\right)$ for all $n \geqslant 1$.

\subsection{Permutation modules}

Let $G$ be a profinite group and $R$ a commutative pseudocompact ring. Let $(X, *)$ be a pointed profinite $G$-space (that is, $X$ is a profinite space on which $G$ acts continuously and such that $g *=* \forall g \in G$ ). Recall (cf. [25, 1.7]) that the free pseudocompact $R$-module on $(X, *)$ is a pseudocompact $R$-module $R[[(X, *)]]$ together with a continuous map $\iota:(X, *) \rightarrow R[(X, *)]$ sending $*$ to 0 and satisfying the following universal property:

Given any pseudocompact $R$-module $M$ and continuous map $\beta: X \rightarrow M$ sending $*$ to 0 , there is a unique continuous homomorphism of $R$-modules $\beta^{\prime}: R[[(X, *)]] \rightarrow M$ such that $\beta^{\prime} \iota=\beta$.

Definition 2.6 (cf. [25, Def. 1.8]). Let $G$ be a profinite group and $R$ a commutative pseudocompact ring. Let $(X, *)$ be a pointed profinite $G$-space. The corresponding pseudocompact permutation module for $G$ is the $R$-module $R[[(X, *)]$ with action from $G$ given by the action of $G$ on $X$ and the universal property of $R[[(X, *)]]$.

Note that the corresponding map $\iota$ is thus $G$-equivariant. The definition can be succinctly stated as follows: a pseudocompact permutation $R[[G]]$-module is a pseudocompact $R[[G]]$-module having a pointed profinite $G$-invariant $R$-basis. By construction, a permutation module is free as an $R$-module, so in particular is a lattice.

Lemma 2.7. The permutation $R[[G]]$-module $R[[(X, *)]]$ is determined by the following universal property:

Given any pseudocompact $R[[G]]$-module $M$ and any continuous $G$-equivariant map $\beta: X \rightarrow M$ sending $*$ to 0 , there is a unique continuous $R[[G]]$-module homomorphism $\left.\beta^{\prime}: R[(X, *)]\right] \rightarrow M$ such that $\beta^{\prime} \iota=\beta$.

Proof. That there exists a unique $R$-module homomorphism is the universal property of $R[[(X, *)]]$ as an $R$-module. Using the universal property again, we see that this map is an $R G$-module homomorphism.

We give several alternative definitions of a permutation module for a profinite group $G$, which in general are not equivalent. Given a profinite group $G$ and an (unpointed) profinite $G$-space $X$, the corresponding discrete permutation module $F(X)$ is defined in 33 to be the module of continuous 
functions $X \rightarrow T$, where $T$ is the maximal submodule in $R$-Mod ${ }^{\mathcal{D}}$ of the injective hull of $R$ as an abstract $R$-module. The pseudocompact permutation module $R[[X]]$ in the sense of [33] is the Pontryagin dual of $F(X)$. This is the same as saying that $R[[X]]$ is the pseudocompact $R[[G]]$-module having profinite $R$-basis $X$. Note that the modules $R[X]$ and $R[(X \cup\{*\}, *)]$ are clearly isomorphic when $X$ is finite. A profinite $G$-space $X$ (resp. profinite pointed $G$-space $(X, *)$ ) can be expressed as the inverse limit of an inverse system of finite (resp. finite pointed) $G$-sets $X_{i}$ (resp. $\left.\left(X_{i}, *\right)\right)$ [27, Lem. 5.6.4]. We have that $R\left[[X] \cong \varliminf_{\varlimsup} R\left[X_{i}\right]\right.$ and $\left.R[(X, *)]\right]=\varliminf_{\varlimsup} R\left[\left(X_{i}, *\right)\right]$. A strict pseudocompact permutation module for $G$ is a module isomorphic to one of the form $\prod_{j \in J} R\left[\left[G / H_{j}\right]\right]$, where $J$ is a set and each $H_{j}$ is a closed subgroup of $G$.

Proposition 2.8. Let $R$ be a commutative local pseudocompact ring with residue class field of characteristic $p$. Let $G$ be a finite p-group and let $U$ be a pseudocompact $R G$-module. The following are equivalent:

1. $U$ is a permutation module,

2. $U$ has a profinite $G$-invariant $R$-basis,

3. $U$ is a strict pseudocompact permutation module,

4. $U$ is an inverse limit of finite rank permutation modules.

Proof. Given a module of the form $\prod_{j \in J} R\left[G / H_{j}\right]$, choose for each $j \in J$ the basis of left cosets of $H_{j}$ in $G$, and let $X$ be the union of these bases (a discrete set) compactified at the point $*$. One now checks that $\left.\prod_{j \in J} R\left[G / H_{j}\right] \cong R[(X, *)]\right]$ by observing that the universal property of Lemma 2.7 follows from the universal property of the product. Thus 3 implies 1 . The equivalence of 3 and 4 follows from 23, Thm $2.2]$ in case $R$ is profinite (note that [23, Cor. 2.3] is not correct as stated, since the module $R[G / H]$ need not be indecomposable when the residue class field of $R$ is not of characteristic $p$ ). The same proof works for pseudocompact $R$ (see final remark of 93 ). As the $G$-space $X$ (resp. pointed $G$-space $(X, *)$ ) is the inverse limit of finite $G$-sets (resp. finite pointed $G$-sets), 2 (resp. 1) implies 4 . We show that 3 implies 2. As $G$ is finite, we may suppose that $U=\prod_{J} R[G / H]$ for some fixed subgroup $H$ of $G$ and discrete set $J$. The result is obvious when $J$ is finite so suppose that $J$ is infinite. Let $J^{*}$ be the one-point compactification of $J$ with point at infinity *. Consider the (unpointed) profinite $G$-space $G / H \times J^{*}$, where $G$ acts only on the left factor, in the obvious way. We obtain

$$
\begin{aligned}
R\left[\left[G / H \times J^{*}\right]\right] & \cong \lim _{F \text { finite }} R[G / H \times(* \cup F)] \\
& \cong \varliminf_{F \text { finite }} R[G / H] \times \prod_{F} R[G / H] \\
& \cong R[G / H] \times \prod_{J} R[G / H] \\
& \cong U \quad \text { (since } J \text { is infinite). }
\end{aligned}
$$

Even for finite groups we can not, in general, suppose that the module $R[G / H]$ is indecomposable, and indeed the class of permutation modules need not be closed under summands. We define an $R$ permutation module to be a summand of a permutation module. For a profinite group $G$, denote by $\operatorname{Perm}(G)$ the module $\prod_{H \leqslant G} R[[G / H]]$ (subgroups of profinite groups are always taken to be closed). A strict pseudocompact $R$-permutation module for $G$ is an object of $\operatorname{Add}^{\mathcal{C}}(\operatorname{Perm}(G))$. The second author showed [33, Cor. 3.21] that every $R$-permutation module for a finite group is strict.

Lemma 2.9. Let $G$ be a finite group and suppose that $|G|$ is not a zero divisor of $R$. If $V$ is a pseudocompact R-permutation module then $H^{1}(H, V)=0$ for all $H \leqslant G$. 
Proof. By the Eckmann-Shapiro Lemma, additivity and the Mackey decomposition formula, we need only check that $H^{1}(H, V)=0$ for $V$ a trivial pseudocompact $R H$-module. But $H^{1}(H, V)$ is isomorphic to group homomorphisms from $H$ to $V$. If $\rho$ is such a homomorphism then for any $h \in H$ we have

$$
0=\rho(1)=\rho\left(h^{|G|}\right)=|G| \rho(h) \Longrightarrow \rho(h)=0
$$

since $|G|$ is not a zero-divisor.

Permutation modules of the form $R[[G / H]]$ can be written as $R \uparrow_{H}^{G}$, the trivial $R H$-module induced up to $G$. A larger class of modules of interest to us is the class of monomial modules (called generalized permutation modules in [36]), namely the category of continuous direct summands of direct products of modules of the form $V \uparrow_{H}^{G}$, where $V$ is an $R$-rank $1 R H$-lattice. When $G$ is finite and $R$ is an integral domain there are only finitely many isomorphism classes of lattices of $R$-rank 1 (because $R$ has only finitely many $|G|$ th roots of unity), so the category of monomial modules is $\operatorname{Add}^{\mathcal{C}}(M)$ with $M=\bigoplus_{H \leqslant G} V_{H} \uparrow G$, where $V_{H}$ runs through the set of isomorphism classes of $R$-rank $1 R H$-lattices. Notice that monomial modules are preserved under induction and restriction.

\section{Change of category}

Recall that $\Lambda-\operatorname{Mod}^{\mathcal{C}}$ has exact inverse limits [18, Ch.IV, Thm 3] and $\Lambda$-Mod ${ }^{\mathcal{D}}$ has exact direct limits. Let $M$ be a finitely generated object of $\Lambda-\operatorname{Mod}^{\mathcal{C}}$ and $E=\operatorname{End}_{\Lambda}(M)$, a pseudocompact algebra by Lemma 2.3. Treating $M$ as a left $E$-module in the obvious way the actions of $\Lambda$ and $E$ are compatible, in so far as $\rho(\lambda m)=\lambda \rho(m)$ for $\rho \in E, \lambda \in \Lambda, m \in M$. In what are perhaps more familiar terms, this amounts to saying that $M$ is a $\Lambda$ - $E^{\mathrm{op}}$-bimodule. Consider the following functors:

$$
\begin{array}{r}
U=\operatorname{Hom}_{\Lambda}(M,-): \Lambda-\operatorname{Mod}^{\mathcal{C}} \rightarrow \operatorname{Mod}^{\mathcal{C}}-E \\
V=-\widehat{\otimes}_{E} M: \operatorname{Mod}^{\mathcal{C}}-E \rightarrow \Lambda-\operatorname{Mod}^{\mathcal{C}} .
\end{array}
$$

Lemma 3.1. The functor $V$ is left adjoint to $U$.

Proof. See the discussion after Lemma 2.3 .

Proposition 3.2. Restricting the domain and codomain of $U$ and $V$ to $\operatorname{Add}^{\mathcal{C}}(M)$ and $\operatorname{Proj}^{\mathcal{C}}-E$ yields mutually inverse equivalences of categories.

Proof. This is more or less standard and the usual proof goes through (see, for instance, 11, Lem. 29.4]). The only difference is that we must check that the functors $U$ and $V$ commute with products rather than sums. But this follows from observations in $\$ 2.2$.

With $M$ still a finitely generated module in $\Lambda-\operatorname{Mod}^{\mathcal{C}}$, we may dualize the functors above to obtain

$$
\begin{array}{r}
U^{\prime}=\operatorname{Hom}_{\Lambda}\left(M,(-)^{*}\right)^{*}=\operatorname{Hom}_{\Lambda}\left(-, M^{*}\right)^{*}: \operatorname{Mod}^{\mathcal{D}}-\Lambda \rightarrow E-\operatorname{Mod}^{\mathcal{D}} \\
V^{\prime}=\left((-)^{*} \widehat{\otimes}_{E} M\right)^{*}=\operatorname{Hom}_{E}(M,-): E-\operatorname{Mod}^{\mathcal{D}} \rightarrow \operatorname{Mod}^{\mathcal{D}}-\Lambda .
\end{array}
$$

Lemma 3.3. The functor $V^{\prime}$ is right adjoint to $U^{\prime}$.

Lemma 3.4. The functor $V$ commutes with direct limits and the functor $V^{\prime}$ commutes with inverse limits.

Proof. These are formal properties of adjoint functors, see for instance [35, Thm 2.6.10].

Again, by duality, restricting the domain and codomain, we obtain an equivalence of categories between $\operatorname{Add}^{\mathcal{D}}\left(M^{*}\right)$ and $E$-Inj ${ }^{\mathcal{D}}$.

These equivalences allow one to deduce properties of $\operatorname{Add}(M)$ from already understood properties of $E$-Proj ${ }^{\mathcal{C}}$ or $\operatorname{Inj}^{\mathcal{D}}-E$. For example, from [18, Ch.III, Cor.1 to Thm 3] it follows that every module in $\operatorname{Add}^{\mathcal{C}}(M)$ is a product of indecomposable summands of $M$. Furthermore, from [18, Ch.IV, Thm.2] this decomposition is essentially unique. Indeed, $\operatorname{Add}^{\mathcal{C}}(M)$ has the exchange property: 
Proposition 3.5 ([18, Ch. IV, Prop. 8]). Let $X$ be a continuous direct summand of the pseudocompact $\Lambda$ module $Y=\prod_{i \in I} Y_{i}$, where each $Y_{i}$ is an indecomposable summand of the finitely generated pseudocompact $\Lambda$-module $M$. There exists a subset $J$ of I such that

$$
Y=X \oplus \prod_{i \in J} Y_{i}
$$

Lemma 3.6 ([9, Lem. A.4]). The functor $V: \operatorname{Mod}^{\mathcal{C}}-E \rightarrow \Lambda-\operatorname{Mod}^{\mathcal{C}}$ commutes with inverse limits.

Proposition 3.7 ([9, Cor. 3.3], [18, Ch.II, Cor.1 to Thm 2]). An inverse limit of projective modules in $\Lambda-\operatorname{Mod}^{\mathcal{C}}$ is again a projective module.

Theorem 3.8. Let $\Lambda$ be a pseudocompact $R$-algebra and $M$ a finitely generated pseudocompact left $\Lambda$ module. The inverse limit in $\Lambda-\operatorname{Mod}^{\mathcal{C}}$ of modules in $\operatorname{Add}^{\mathcal{C}}(M)$ is again an object of $\operatorname{Add}^{\mathcal{C}}(M)$.

Proof. The proof of [23, Thm 2.2] works in this greater generality, but this also follows easily from the results above: an inverse system in $\operatorname{Add}^{\mathcal{C}}(M)$ yields, by applying $U$, an inverse system of projective modules, whose limit is projective by Proposition 3.7 Applying $V$ to this limit we obtain an object of $\operatorname{Add}^{\mathcal{C}}(M)$. But $V$ commutes with inverse limits by Lemma 3.6, and hence this module is the inverse limit of the original inverse system.

Remark: This result is false without the topology. In 4, Bergman shows that any abstract module can be expressed as an inverse limit of injective modules. This is most striking when $\Lambda$ is self-injective (for instance, when $R$ is a field and $\Lambda$ is the group algebra of a finite group) [3, §1.6]. In this case projective modules are the same as injective modules, so that every abstract module is an inverse limit of projective modules.

Remark: Let $R$ be a finite unramified extension of the $p$-adic integers $\mathbb{Z}_{p}$ and let $G$ be a finite group. When $G$ is cyclic of order $p$ or $p^{2}$ it is known that there are only finitely many isomorphism classes of finitely generated indecomposable $R G$-lattices $[15$, Thm 33.7, Cor. 33.3a]. It follows that for general finite $G$ there are finitely many isomorphism classes of indecomposable finitely generated $R G$-lattices that are projective relative to subgroups of order $p$ or $p^{2}$ (for the definition of relative projectivity see after Lemma 6.11, for further details see for example [3, §3.6], or [22] for profinite modules). Denote by $M_{G}$ the sum of (a representative of each isomorphism class of) the indecomposable $R G$-lattices projective relative to cyclic subgroups of order $p$ or $p^{2}$. If $G$ is cyclic of order $p$ or $p^{2}$, the category add $\left(M_{G}\right)$ coincides with the category of finitely generated $R G$-lattices. As pseudocompact $R G$-lattices are inverse limits of finite rank $R G$-lattices by Lemma 2.1. Theorem 3.8 tells us that compact $R G$-lattices are summands of products of indecomposables, hence products of indecomposables by the exchange property (Proposition 3.5). For general $G$, a compact $R G$-lattice $L$ that is projective relative to cyclic subgroups of order $p$ or $p^{2}$ is a direct summand of

$$
\prod_{\substack{C \leqslant G \\ C \cong C_{p} \text { or }}} L \downarrow_{p^{2}} \uparrow^{G}
$$

and hence (by the theorem) is an object of $\operatorname{Add}\left(M_{G}\right)$.

Remark: Every profinite $G$-set $X$ can be expressed as an inverse limit of finite $G$-sets $X=\lim _{\longleftarrow} X_{i}$ [27, Lem. 5.6.4(a)] and hence $R[[X]]=\lim R\left[X_{i}\right]$. Theorem 3.8 now yields a different proof of the result of 33, Corol. 3.21]: a pseudocompact permutation module can be expressed as a summand of a product of modules isomorphic to $R[G / H](H \leqslant G)$.

\section{Other limits}

We have observed that $\Lambda-\operatorname{Mod}^{\mathcal{C}}$ has exact inverse limits and dually that $\Lambda$-Mod ${ }^{\mathcal{D}}$ has exact direct limits. It is interesting to note that $\Lambda-\operatorname{Mod}^{\mathcal{C}}$ also has direct limits and $\Lambda$-Mod ${ }^{\mathcal{D}}$ has inverse limits (though of course, they are not exact) [18, Ch.II, Cor. 2 to Thm 1]. Inverse limits in $\Lambda$-Mod ${ }^{\mathcal{D}}$ are obtained by taking 
the inverse limit in $\Lambda$-Mod ${ }^{\text {abs }}$, giving it the discrete topology and then taking the submodule generated by elements annihilated by an open ideal of $\Lambda$. Direct limits in $\Lambda-\mathrm{Mod}^{\mathcal{C}}$ are described in [14, §2]. Note that these limits do not in general commute with restriction to subgroups.

By definition, $\Lambda=\lim \Lambda / I_{j}$ for some set of open ideals $I_{j}$ of $\Lambda$ with each $\Lambda / I_{j}$ an $R$-algebra of finite length over $R$. Given such an ideal $I$ and a module $M$ (compact, discrete torsion or abstract), denote by $T^{I} M$ the set $\{m \in M \mid I m=0\}$. Note that $T^{I} \varliminf^{\mathcal{D}}=\lim ^{\mathcal{D}} T^{I}$. Let $T M$ denote the union of the $T^{I} M$ as $I$ varies over the open ideals of $\Lambda$.

Proposition 4.1. A direct limit in $\Lambda-\mathrm{Mod}^{\mathcal{C}}$ of projective modules in $\Lambda-\bmod ^{\mathcal{C}}$ is again projective. An inverse limit in $\Lambda-\mathrm{Mod}^{\mathcal{D}}$ of injective modules in $\Lambda-\bmod ^{\mathcal{D}}$ is again injective.

Proof. We prove the second statement. First suppose that $\Lambda$ has finite length over $R$. An inverse system in $\operatorname{add}^{\mathcal{D}}\left(\Lambda^{*}\right)$ can be treated as an inverse system in $\operatorname{add}^{\mathcal{C}}\left(\Lambda^{*}\right)$, and so by Theorem 3.8 it has an inverse limit in $\operatorname{Add}^{\mathcal{C}}\left(\Lambda^{*}\right)$. The module obtained by giving this module the discrete topology coincides with the inverse limit in $\Lambda$-Mod ${ }^{\mathcal{D}}$ because all these modules are torsion.

In $\operatorname{Add}^{\mathcal{C}}\left(\Lambda^{*}\right)$ our module has the form $\bigoplus I_{j} \widehat{\otimes}_{R} V_{j}$, where $I_{j}$ runs through the indecomposable summands of $\Lambda^{*}$ and $V_{j}$ is a free module in $R$-Mod ${ }^{\mathcal{C}}$. But each $I_{j}$ is of finite length, hence finitely presented, so that $I_{j} \widehat{\otimes} V_{j} \cong I_{j} \otimes V_{j}$ by Proposition 2.2 If we ignore the topology, $V_{j}$ is a free abstract module, by 10. Thm 3.3]. Thus, the module is in $\operatorname{Add}^{\mathcal{D}}\left(\Lambda^{*}\right)=\operatorname{Inj}^{\mathcal{D}}-\Lambda$.

For the general case, write $\Lambda=\lim \Lambda / I_{i}$. Denote by $X=\lim _{\longleftarrow} X_{j}$ our module, where each $X_{j}$ is injective. Then

$$
T^{I_{i}} \lim ^{\mathcal{D}} X_{j}=\lim ^{\mathcal{D}} T^{I_{i}} X_{j} .
$$

But each $T^{I_{i}} X_{j}$ is injective as a $\Lambda / I_{i}$-module, so by the special case above $\lim _{j}^{\mathcal{D}} T^{I_{i}} X_{j}$ is in $\operatorname{Inj}^{\mathcal{D}}-\Lambda / I_{i}$. Now take $\lim _{I_{i}}$, observing that by [18, Ch.2, Cor. 1 to Thm 1] a direct limit of modules injective over $\Lambda / I_{i}$ is itself injective over $\Lambda$.

Theorem 4.2. A direct limit in $\Lambda-\operatorname{Mod}^{\mathcal{C}}$ of modules in $\operatorname{add}^{\mathcal{C}}(M)$ is in $\operatorname{Add}^{\mathcal{C}}(M)$. An inverse limit in $\Lambda$-Mod ${ }^{\mathcal{D}}$ of modules in $\operatorname{add}^{\mathcal{D}}\left(M^{*}\right)$ is in $\operatorname{Add}^{\mathcal{D}}\left(M^{*}\right)$.

Proof. We prove the first statement. Let $X=\lim _{i} X_{i}$ be a direct limit of modules in $\operatorname{add}^{\mathcal{C}}(M)$. Then by Proposition 3.2 there are projective right $E$-modules $P_{i}$ for which

$$
X=\lim _{\longrightarrow} X_{i}=\lim _{\longrightarrow} V\left(P_{i}\right)=V\left(\lim _{\longrightarrow} P_{i}\right),
$$

where the last equality is from Lemma 3.4. Hence $X \in \operatorname{Add}^{\mathcal{C}}(M)$ by Propositions 4.1 and 3.2 .

\section{$5 \quad$ Flatness}

We say that a module $X$ in $\Lambda$-Mod ${ }^{\mathcal{C}}$ is flat if the functor $-\widehat{\otimes}_{\Lambda} X$ is exact on $\operatorname{Mod}^{\mathcal{C}}-\Lambda$. A module $X$ in $\Lambda$-Mod ${ }^{\mathcal{C}}$ is flat if, and only if, it is projective. This follows from the existence of projective covers, and so the usual proof for perfect rings (see eg. 20, Thm 24.25]) carries through.

We consider other criteria for flatness. Observe that a direct limit of projective modules need not be projective, but by Proposition 4.1 a direct limit in $\Lambda-\mathrm{Mod}^{\mathcal{C}}$ of finitely generated projectives is again projective. The converse is false: a projective module need not be a direct limit of finitely generated projectives. This is easily seen in the dual case, by taking $\Lambda=R=k$, a finite field. The sum $\bigoplus_{n \in \mathbb{N}} k$ of countably many copies of $k$ (an injective object of $\operatorname{Mod}^{\mathcal{D}}-k$ ) is countable, hence not an inverse limit of finite dimensional vector spaces by [27, Prop. 2.3.1(b)].

On the other hand, if we allow summands, the problem vanishes: If $X$ is an object of $\operatorname{Inj}^{\mathcal{D}}-\Lambda$ then $X=\bigoplus_{j \in J} I_{j}$ with each $I_{j}$ an indecomposable injective. Consider $Y=\prod_{j \in J} I_{j}$ in the category of abstract $\Lambda$-modules. Then $X \subseteq T Y \subseteq Y$. Because $Y$ is an inverse limit of finitely cogenerated injectives, it follows that $T Y$ is an inverse limit in $\operatorname{Ind}^{\mathcal{D}}-\Lambda$. The inclusion $X \rightarrow T Y$ splits because $X$ is injective. 


\section{$6 \operatorname{Add}(M)$ precovers and covers}

It is convenient to follow the approach to covers and precovers given in [17. In this section, $M$ denotes a finitely generated module in $\Lambda-\operatorname{Mod}^{\mathcal{C}}$.

Definition 6.1 (cf. [17, §5]). Let $X$ be an object of $\Lambda-\mathrm{Mod}^{\mathcal{C}}$. The continuous homomorphism $\rho: P \rightarrow X$ is an $\operatorname{Add}(M)$-precover of $X$ if

- $P \in \operatorname{Add}(M)$,

- Given any continuous homomorphism $\alpha: S \rightarrow X$ with $S$ in $\operatorname{Add}(M)$, there exists a continuous homomorphism $\gamma: S \rightarrow P$ such that $\rho \gamma=\alpha$.

An $\operatorname{Add}(M)$-precover $\rho: P \rightarrow X$ is an $\operatorname{Add}(M)$-cover of $X$ if every continuous homomorphism $\gamma: P \rightarrow P$ such that $\rho \gamma=\rho$ is an automorphism of $P$.

The $\operatorname{Add}(M)$-cover of $X$, if it exists, is clearly unique up to isomorphism. It is also easily checked that the $\operatorname{Add}(M)$-cover of $X$ is a direct summand of any $\operatorname{Add}(M)$-precover of $X$, and that an $\operatorname{Add}(M)$ precover is a split-surjection if, and only if, $X \in \operatorname{Add}(M)$. It follows that an $\operatorname{Add}(\Lambda)$-cover corresponds to the usual notion of a projective cover - that is, an $\operatorname{Add}(\Lambda)$-cover $\rho: P \rightarrow X$ of $X$ is precisely a surjective homomorphism from the projective $\Lambda$-module $P$ with $P$ minimal with respect to direct sum decompositions (cf. [3, §1.5]).

Theorem 6.2. Let $M$ be a finitely generated $\Lambda$-module and let $X$ be any pseudocompact $\Lambda$-module. The $\operatorname{Add}(M)$-cover of $X$ exists.

Proof. Denote by $\varepsilon, \eta$ the counit and unit of the adjunction of Lemma 3.1 By results mentioned there, restricting the domain and codomain of $\varepsilon$ to $\operatorname{Add}(M), \operatorname{Proj}(E)$ yields a natural isomorphism. Since $E$ is pseudocompact by Lemma 2.3. $\operatorname{Mod}^{\mathcal{C}}-E$ has projective covers.

Let $\rho: P \rightarrow U(X)$ be the projective cover of $U(X)$. Then $V(P)$ is an $\operatorname{Add}(M)$-module and

$$
\varepsilon_{X} \circ V(\rho): V(P) \rightarrow V U(X) \rightarrow X
$$

is the $\operatorname{Add}(M)$-cover of $X$. We sketch the argument. Given $\alpha: S \rightarrow X$ with $S \in \operatorname{Add}(M)$, there is a homomorphism $\gamma: U(S) \rightarrow P$ such that $\rho \gamma=U(\alpha)$ because $\rho$ is a precover. The diagram

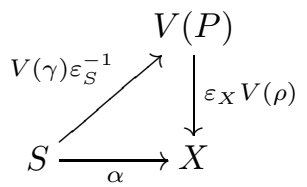

commutes:

$$
\begin{aligned}
\varepsilon_{X} V(\rho) V(\gamma) \varepsilon_{S}{ }^{-1} & =\varepsilon_{X} V(\rho \gamma) \varepsilon_{S}{ }^{-1} \\
& =\varepsilon_{X} V U(\alpha) \varepsilon_{S}{ }^{-1} \\
& =\alpha \varepsilon_{S} \varepsilon_{S}{ }^{-1} \\
& =\alpha
\end{aligned}
$$

hence $\varepsilon_{X} V(\rho)$ is a precover. To check it is a cover, consider $\gamma$ completing the following diagram:

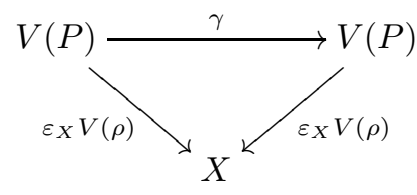

One checks that $\eta_{P}{ }^{-1} U(\gamma) \eta_{P}: P \rightarrow P$ is an isomorphism using the counit-unit equations and the fact that $\rho$ is a cover. Hence $\gamma$ too is an isomorphism. 
From the construction it follows that if $X$ is finitely generated over $\Lambda$, then so is its $\operatorname{Add}(M)$-cover. In the following definition, $H \leqslant{ }_{G} G$ indicates that $H$ runs through a set of representatives of the classes of $G$-conjugates of subgroups of $G$.

Definition 6.3. Let $G$ be a finite group and $X$ a pseudocompact $R G$-module. A permutation precover of $X$ is an $\operatorname{Add}(M)$-precover of $X$, where

$$
M=\prod_{H \leqslant G G} R[G / H] .
$$

The permutation cover of $X$ is the corresponding $\operatorname{Add}(M)$-cover.

Let $R$ be pseudocompact integral domain. A monomial precover (or generalized permutation precover) of $X$ is an $\operatorname{Add}(M)$-precover of $X$, where

$$
M=\prod_{H \leqslant G G} V_{H} \uparrow_{H}^{G}
$$

and $V_{H}$ runs through the set of isomorphism classes of $R$-rank $1 R H$-lattices. The monomial cover of $X$ is the corresponding $\operatorname{Add}(M)$-cover.

Both the permutation and monomial covers of $X$ exist by Theorem 6.2

We present some useful properties of permutation and monomial precovers that we will require in Section 8. Following Samy Modeliar [29, we say that an $R G$-module homomorphism $\rho: V \rightarrow U$ is supersurjective if the induced homomorphism $\rho^{H}: V^{H} \rightarrow U^{H}$ is surjective for every subgroup $H$ of $G$. Similarly, $\rho$ is monomial supersurjective if the induced homomorphism $\operatorname{Hom}_{R G}(W \uparrow G, V) \rightarrow$ $\operatorname{Hom}_{R G}\left(W \uparrow_{H}^{G}, U\right)$ is surjective for every $R$-rank $1 R H$-lattice $W$. Note that monomial supersurjective homomorphisms are supersurjective.

Lemma 6.4. 1. A homomorphism from a permutation module is a permutation precover if, and only if, it is supersurjective.

2. Let $\pi: X \rightarrow Y$ be supersurjective and $f: L \rightarrow Y$ a homomorphism from a permutation module $L$. Then $f$ lifts to a homomorphism $\tilde{f}: L \rightarrow X$ such that $\pi \tilde{f}=f$.

Proof. 1. Given a subgroup $H$ of $G$, we have isomorphisms of functors

$$
\operatorname{Hom}_{R G}\left(R \uparrow_{H}^{G},-\right) \cong \operatorname{Hom}_{R H}\left(R,(-) \downarrow_{H}\right) \cong(-)^{H} .
$$

Suppose first that $\rho: P \rightarrow U$ is a permutation precover. Via the above isomorphisms, an element $u \in U^{H}$ is $\alpha(H)$ for a homomorphism $\alpha: R[G / H] \rightarrow U$. This homomorphism factors as $\alpha=\rho \gamma$ for a homomorphism $\gamma: R[G / H] \rightarrow P$ and hence $\gamma(H)$ is an element of $P^{H}$ mapping onto $u$ via $\rho^{H}$, showing that $\rho^{H}$ is surjective.

Suppose now that $\rho$ is supersurjective. An $R$-permutation module is a finite sum of isotypic components so it suffices to check that we can lift a homomorphism $\alpha: X \rightarrow U$ where $X$ is a direct summand of $\prod R[G / H]$ for some subgroup $H$ of $G$. Let $\iota: X \rightarrow \prod R[G / H], \pi: \prod R[G / H] \rightarrow X$ be the splitting maps. By the functor isomorphisms above and supersurjectivity, there is a homomorphism $\gamma: \prod R[G / H] \rightarrow P$ such that $\rho \gamma=\alpha \pi$, and now $\gamma \iota$ is the required lifting of $\alpha$.

2. Let $\alpha: P \rightarrow X$ be a permutation precover of $X$. Then $\pi \alpha$ is supersurjective, hence a permutation precover of $Y$. It follows that there exists a $\gamma: L \rightarrow P$ such that $\pi \alpha \gamma=f$, and now $\alpha \gamma=\widetilde{f}$ is the required lift.

In just the same way we have:

Lemma 6.5. Let $R$ be a pseudocompact integral domain. 
1. A homomorphism from a monomial module is a monomial precover if, and only if, it is monomial supersurjective.

2. Let $\pi: X \rightarrow Y$ be monomial supersurjective and $f: L \rightarrow Y$ a homomorphism from a monomial module $L$. Then $f$ lifts to a homomorphism $\widetilde{f}: L \rightarrow X$ such that $\pi \widetilde{f}=f$.

Lemma 6.6. If $M$ is an $R$-permutation module and $\rho: L \rightarrow M$ is supersurjective, then $\rho$ splits.

Proof. Let $\gamma: C \rightarrow L$ be a permutation precover of $L$. The composition $\rho \gamma$ is supersurjective and hence a permutation precover of the $R$-permutation module $M$. Thus $\rho \gamma$ splits, hence so does $\rho$.

A lattice $L$ in $R G-\operatorname{Mod}^{\mathcal{C}}$ is said to be coflasque if $H^{1}(H, L)=0$ for all $H \leqslant G$.

Proposition 6.7. Let $0 \rightarrow L \rightarrow M \stackrel{\rho}{\rightarrow} N \rightarrow 0$ be a short exact sequence of RG-lattices. Suppose that $N$ is an R-permutation module and that $L$ is coflasque. Then the sequence splits.

Proof. For any subgroup $H$ of $G$ the sequence $0 \rightarrow L^{H} \rightarrow M^{H} \stackrel{\rho^{H}}{\longrightarrow} N^{H} \rightarrow 0$ is exact because $H^{1}(H, L)=$ 0 . Thus $\rho$ is supersurjective and hence split by Lemma 6.6.

Corollary 6.8. Let $0 \rightarrow L \rightarrow M \rightarrow N \rightarrow 0$ be a short exact sequence of RG-modules with $L, N$ both $R$-permutation modules. If $|G|$ is not a zero divisor of $R$, then the sequence splits.

Proof. By Lemma 2.9 $L$ is coflasque, so the result is immediate from the above proposition.

We will give a formula for the permutation cover of a pseudocompact $R G$-module using methods similar to those developed by Samy Modeliar [30, when $R$ is a complete discrete valuation ring whose residue class field has characteristic $p$ and $G$ is a finite group. Let $\pi$ be a prime element of $R$. Given a pseudocompact $R G$-module $X$ and a subgroup $H$ of $G$, consider the map

$$
\begin{aligned}
\operatorname{Tr}_{H}^{G}: X^{H} & \rightarrow X^{G} \\
x & \mapsto \sum_{g \in G / H} g x
\end{aligned}
$$

where $g$ runs through a set of left coset representatives of $G / H$. This is a special case of the map defined in [3, Def. 3.6.2] and the properties given there apply. Using [3, Lem. 3.6.3] one sees that if $|G / H|$ is coprime to $p$, then $\operatorname{Tr}_{H}^{G}$ is surjective.

Definition 6.9. Given a p-subgroup $P$ of $G$ and a pseudocompact $R G$-module $X$, define

$$
\begin{gathered}
X^{[P]}:=\frac{X^{P}}{\sum_{Q<P} \operatorname{Tr}_{Q}^{P}\left(X^{Q}\right)+\pi X^{P}} \\
X^{[P]]}:=\frac{X^{P}}{\sum_{\substack{P<Q \\
Q \text { group }}} X^{Q}+\sum_{Q<P} \operatorname{Tr}_{Q}^{P}\left(X^{Q}\right)+\pi X^{P}}
\end{gathered}
$$

wherein the symbol $<$ indicates strict inclusion.

Note that in $X^{[P]}$ and $X^{\llbracket P \rrbracket]}$ we quotient out by $\pi X^{P}$, so that both are $(R / \pi)\left[\mathrm{N}_{G}(P) / P\right]$-modules. The module $X^{[P]}$ is studied in [7] and references therein, while $X^{\llbracket P \rrbracket}$ is inspired by [30, Def. 5]. An $R G$ module homomorphism $f: X \rightarrow Y$ sends $X^{P}$ into $Y^{P}$ and the denominator of $X^{[P]}$ into the denominator of $Y^{[P]}$, and hence induces a natural map $f^{[P]}: X^{[P]} \rightarrow Y^{[P]}$. Similarly, $f$ induces a natural map $f^{\llbracket P \rrbracket}: X^{\llbracket P \rrbracket} \rightarrow Y^{\llbracket P \rrbracket}$.

Remark: Note that $X^{[P]} \downarrow_{P / P}=\left(X \downarrow_{P}\right)^{[P]}$. Such a strong statement cannot be made for $X^{[P \rrbracket}$ but we do at least have that $X\left[\left[P \rrbracket=\left(X \downarrow_{\mathrm{N}_{G}(P)}\right)[[P \rrbracket]\right.\right.$ because if $Q$ is a $p$-subgroup of $G$ properly containing $P$, then so is $Q \cap \mathrm{N}_{G}(P)=\mathrm{N}_{Q}(P)$. 
Lemma 6.10. The homomorphism $f: M \rightarrow N$ in $R G$-Mod ${ }^{\mathcal{C}}$ is supersurjective if, and only if, $f \llbracket P \rrbracket$ : $M^{[[P]]} \rightarrow N^{[[P]]}$ is surjective for every p-subgroup $P$ of $G$.

Proof. The forward implication is clear. If $f^{H}$ is not surjective for some subgroup $H$ of $G$, one easily checks using the surjectivity of $\operatorname{Tr}_{P}^{H}$ that $f^{P}$ is also not surjective for $P$ any Sylow $p$-subgroup of $H$. Thus $f$ is supersurjective if, and only if, $f^{P}$ is surjective for every $p$-subgroup of $G$. Note further that if $f^{P}$ is surjective for every subgroup $P$ contained in a fixed Sylow $p$-subgroup $S$ of $G$, then $f$ is supersurjective (since $M^{g} P=g M^{P}$ ). Thus we restrict once and for all to a Sylow $p$-subgroup $S$ of $G$.

Given a $p$-subgroup $P$ of $S$, we will show that

$$
f^{[P \rrbracket} \text { surjective } \forall P \Longrightarrow f^{[P]} \text { surjective } \forall P \Longrightarrow f^{P} \text { surjective } \forall P \text {. }
$$

We prove the second implication by induction on the order of $P$. The base case $P=1$ is Nakayama's Lemma. Fix a $p$-subgroup $P$ of $S$ and suppose by induction that $f^{Q}$ is surjective for every proper subgroup $Q$ of $P$. As $f^{[P]}$ is surjective, we can write a given $n \in N^{P}$ as $f(m)+\sum_{Q<P} \operatorname{Tr}_{Q}^{P}\left(n_{Q}\right)+\pi x$ for some $m \in M^{P}, n_{Q} \in N^{Q}$ and $x \in N^{P}$. But $n_{Q}=f\left(m_{Q}\right)$ for some $m_{Q} \in M^{Q}$ by induction, and hence

$$
n-\pi x=f(m)+\sum_{Q<P} \operatorname{Tr}_{Q}^{P}\left(f\left(m_{Q}\right)\right)=f\left(m+\sum_{Q<P} \operatorname{Tr}_{Q}^{P}\left(m_{Q}\right)\right) \in f\left(M^{P}\right) .
$$

This shows that $f^{P}$ is surjective modulo $\pi$, so that $f^{P}$ is surjective by Nakayama's Lemma. We prove the first implication by induction on the index of $P$ in $S$, the case $P=S$ being trivial. Consider the cokernel $C$ of $f^{[P]}$. Then $C^{[P]]}$ is the cokernel of $f^{[P]]}$, so it is 0 by hypothesis. We will show that $C_{\mathrm{N}_{S}(P)}=0$, which implies that $C=0$ since taking coinvariants by a $p$-group cannot kill a non-zero module. We must check that

$$
\sum_{\substack{P<Q \\ Q p-\text { group }}} C^{Q} \leqslant \sum_{Q<P} \operatorname{Tr}_{Q}^{P}\left(C^{Q}\right)
$$

in $C_{\mathrm{N}_{S}(P)}$. Consider $c \in C^{Q}$. We may suppose without loss of generality that $|Q: P|=p$. By induction, $c=\sum \operatorname{Tr}_{T}^{Q}\left(d_{T}\right)$ with $d_{T} \in C^{T}$ (T a proper subgroup of $Q$ ). Fix such a $d=d_{T}$ and suppose without loss of generality that $|Q: T|=p$. Whenever $A \leqslant B$ are subgroups, denote by $\operatorname{Res}_{A}^{B}: C^{B} \rightarrow C^{A}$ the inclusion. The Mackey formula yields

$$
\operatorname{Res}_{P}^{Q} \operatorname{Tr}_{T}^{Q}(d)=\sum_{q \in P \backslash Q / T} \operatorname{Tr}_{P \cap{ }^{q} T}^{P} q \operatorname{Res}_{P^{q} \cap T}^{T} d .
$$

If $P \neq T$ then, since both are normal in $Q$, no conjugate of $T$ is equal to $P$ and hence the corresponding element is induced from proper subgroups of $P$ as required. We are left with the case where $P=T$. The sum simplifies to

$$
\operatorname{Res}_{P}^{Q} \operatorname{Tr}_{T}^{Q}(d)=\sum_{q \in Q / P} q d .
$$

But $Q \subseteq \mathrm{N}_{S}(P)$ and hence $\sum_{q \in Q / P} q d$ is equal to $p d$ in $C_{N_{S}(P)}$, so is 0 .

Lemma 6.11. Let $G$ be a finite group and $P, Q$ p-subgroups of $G$. If $P$ is not $G$-conjugate to $Q$, then

$$
R[G / P]^{[Q]]}=0 .
$$

Proof. By the remark before Lemma 6.10 and the Mackey decomposition, we may restrict to $\mathrm{N}_{G}(Q)$ and hence suppose that $Q$ is normal in $G$. There are two cases:

- $P \cap Q<Q$. The module $R[G / P] \downarrow_{Q}$ is a direct sum of modules of the form $R\left[Q /{ }^{g} P \cap Q\right]$. But ${ }^{g} P \cap Q<Q$ and hence $R[G / P]^{[Q]}=0$ by the remark, so that $R[G / P]^{[Q]]}=0$ also.

- $P \cap Q=Q$, so that $Q<P$. For any $g \in G$ the basis element $g P$ is in $R[G / P]^{g} P$, hence $g P=0$ in $R[G / P]^{[[Q]}$ because $Q<{ }^{g} P$. 
Here and in Section 8 we make use of the concept of relative projectivity. Recall that an $R G$-module $U$ is projective relative to the subgroup $H$ if any continuous epimorphism of $R G$-modules $V \rightarrow U$ that splits as an $R H$-module homomorphism, splits as an $R G$-module homomorphism. This is equivalent to saying that $U$ is a direct summand of an $R H$-module induced up to $G$, or to saying that the identity map of $U$ can be written as $\operatorname{Tr}_{H}^{G}(\alpha):=\sum_{x \in G / H} x \alpha x^{-1}$ for some continuous $R H$-endomorphism $\alpha$ of $U$ (the proof of [3, Prop. 3.6.4] goes through for pseudocompact modules). Dually, a module $U$ is injective relative to the subgroup $H$ if any continuous monomorphism of $R G$-modules $U \rightarrow V$ that splits as an $R H$-module homomorphism, splits as an $R G$-module homomorphism.

If $U$ is an indecomposable finitely generated $R G$-module, a subgroup $Q$ of $G$ minimal with respect to the condition that $U$ is relatively $Q$-projective is called a vertex of $U$. The vertices of $U$ are $p$-subgroups and are conjugate in $G$ [3, Prop. 3.10.2]. Furthermore, there is an indecomposable finitely generated $R Q$ module $S$ (unique up to conjugation in $\mathrm{N}_{G}(Q)$ ) such that $U \mid S \uparrow^{G}$, called a source of $U$ (the notation $X \mid Y$ indicates that the module $X$ is isomorphic to a direct summand of $Y$ ). If $U$ has source the trivial module $R$, we call $U$ a trivial source module. Given a finitely generated indecomposable $R\left[\mathrm{~N}_{G}(P)\right]$-lattice $E$ with vertex $P$, denote by $M(P, E)$ its Green correspondent [3, Sec. 3.12]. Thus $M(P, E)$ is an indecomposable $R G$-lattice with vertex $P$ such that $M(P, E) \mid E \uparrow^{G}$ and $E \mid M(P, E) \downarrow_{\mathrm{N}_{G}(P)}$. Furthermore, $M(P, E)$ is the only summand of $E \uparrow^{G}$ with vertex $P$ and $E$ is the only summand of $M(P, E) \downarrow_{\mathrm{N}_{G}(P)}$ with vertex $P$, the other summands of $E \uparrow^{G}$ being projective relative to strictly smaller subgroups and the other summands of $M(P, E) \downarrow_{\mathrm{N}_{G}(P)}$ being projective relative to proper conjugates of $P$ [3, Thm 3.12.2]. More generally, when $E$ is a product of finitely generated indecomposable $R\left[\mathrm{~N}_{G}(P)\right]$-modules with vertex $P$, denote by $M(P, E)$ the product of the corresponding Green correspondents. Note that $E$ is still a (continuous) summand of $M(P, E) \downarrow_{\mathrm{N}_{G}(P)}$ and $M(P, E)$ is still a (continuous) summand of $E \uparrow G$ - this can be seen by direct calculation with the product topology.

Let $E$ be a product of indecomposable trivial source $R\left[\mathrm{~N}_{G}(P)\right]$-modules with vertex $P$ and let the $R G$-module $M(P, E)$ be as above. Let $j: M(P, E) \rightarrow E \uparrow^{G}$ be a split inclusion and define further the split inclusion $s: E \rightarrow E \uparrow{ }^{G} \downarrow_{\mathrm{N}_{G}(P)}$ by $s(e)=1 \widehat{\otimes} e$.

Lemma 6.12. 1. The maps $j^{[P]}, s^{[P]}$ are isomorphisms.

2. If $M$ is an $R G$-module and $f: E \rightarrow M \downarrow_{\mathrm{N}_{G}(P)}$ is an $R\left[\mathrm{~N}_{G}(P)\right]$-module homomorphism, there exists an $R G$-module homomorphism $\widehat{f}: M(P, E) \rightarrow M$ such that

$$
\widehat{f}^{[P]}=f^{[P]} \gamma
$$

where $\gamma$ is an isomorphism from $M(P, E)^{[P]}$ to $E^{[P]}=E / \pi E$.

Proof. 1. We show the result for $s$, the argument for $j$ being similar. Let $E \uparrow{ }^{G} \downarrow_{\mathrm{N}_{G}(P)}=s(E) \oplus Y$. To prove the claim, it is sufficient to show that $Y^{[P]}=0$. By the Green correspondence, an indecomposable summand of $Y$ is a direct summand of a module of the form $R\left[\mathrm{~N}_{G}(P) / H\right]$ for $H$ a subgroup of some proper conjugate ${ }^{g} P$ of $P$. We have

$$
R\left[\mathrm{~N}_{G}(P) / H\right]^{[P]} \downarrow_{P}=\left(R \uparrow_{H}^{\mathrm{N}_{G}(P)} \downarrow_{P}\right)^{[P]} \cong \bigoplus_{x \in P \backslash \mathrm{N}_{G}(P) / H}\left(R \downarrow_{x_{H \cap P} \uparrow^{P}}\right)^{[P]}=0,
$$

because each ${ }^{x} H \cap P \subseteq{ }^{g} P \cap P$ is a proper subgroup of $P$.

2. The map $f$ extends by Frobenius reciprocity to a unique $R G$-module homomorphism $\tilde{f}: E \uparrow^{G} \rightarrow M$ given on pure tensors by $\widetilde{f}(g \widehat{\otimes} e)=g f(e)$. Note that $\widetilde{f} s=f$. Define $\widehat{f}=\widetilde{f} j$. We have

$$
\widehat{f}^{[P]}=\widetilde{f}^{[P]} j^{[P]}=\widetilde{f}^{[P]} s^{[P]}\left(\left(s^{[P]}\right)^{-1} j^{[P]}\right)=f^{[P]} \gamma,
$$

where $\gamma=\left(s^{[P]}\right)^{-1} j^{[P]}$. 
Let $X$ be a pseudocompact $R G$-module. Given a $p$-subgroup $P$ of $G$, let $\varphi_{P}: E_{P} \rightarrow X \llbracket[P \rrbracket$ be the projective cover of $X^{\llbracket P \rrbracket}$ as an $R\left[\mathrm{~N}_{G}(P) / P\right]$-module. By projectivity of $E_{P}, \varphi_{P}$ lifts to a homomorphism $E_{P} \rightarrow X^{P}$. Composing with the inclusion we obtain a homomorphism $\theta_{P}: E_{P} \rightarrow X \downarrow_{\mathrm{N}_{G}(P)}$, which by Lemma 6.12 yields an $R G$-module homomorphism $\widehat{\theta_{P}}: M\left(P, E_{P}\right) \rightarrow X$ such that $\widehat{\theta_{P}}{ }^{[P]}=\theta_{P}^{[P]} \gamma_{P}$ for some isomorphism $\gamma_{P}: M\left(P, E_{P}\right)^{[P]} \rightarrow E_{P}^{[P]}$. Note that $\varphi_{P}^{[\llbracket P \rrbracket]} \gamma_{P}^{\llbracket P] \rrbracket}=\theta_{P}^{[P \rrbracket} \gamma_{P}^{\llbracket P \rrbracket]}={\widehat{\theta_{P}}}^{\llbracket P \rrbracket]}$. Denote by

$$
\theta: C=\prod_{P} M\left(P, E_{P}\right) \rightarrow X
$$

the homomorphism that acts as $\widehat{\theta_{P}}$ on the factor $M\left(P, E_{P}\right)$, as $P$ ranges through a set of representatives of the $G$-conjugacy classes of $p$-subgroups of $G$. For each $P$, denote by $m_{P}: M\left(P, E_{P}\right) \rightarrow C$ the inclusion, noting that $m_{P}^{\llbracket P \rrbracket}$ is an isomorphism by Lemma 6.11,

Theorem 6.13. The map $\theta$ is a permutation cover of $X$.

Proof. By Lemmas 6.4 and 6.10, to check that $\theta$ is a precover we must check that $\theta^{[P \rrbracket}: C^{\llbracket P \rrbracket]} \rightarrow X^{\llbracket P \rrbracket}$ is surjective for any $p$-subgroup $P$ of $G$. But

$$
\theta^{[[P]]} m_{P}^{[[P]]}={\widehat{\theta_{P}}}^{[[P]]}=\varphi_{P}^{[P] \rrbracket} \gamma_{P}^{[P]]},
$$

and hence $\theta^{\llbracket P \rrbracket}$ is surjective.

It remains to check that $\theta$ is a cover. If not, there exists a permutation precover $d: D \rightarrow X$ and a non-surjective homomorphism $f: D \rightarrow C$ such that $\theta f=d$. By Lemma 6.10 there is a $p$-subgroup $P$ such that $f^{\llbracket P \rrbracket}: D^{\llbracket P \rrbracket]} \rightarrow C^{\llbracket P \rrbracket}$ is not surjective. For this $P$, let $\rho: S \rightarrow D^{\llbracket P \rrbracket}$ be a projective cover of $D^{\llbracket P \rrbracket}$ as an $R\left[\mathrm{~N}_{G}(P) / P\right]$-module. By the projectivity of $S$ we obtain a map $\delta$ yielding the following commutative diagram:

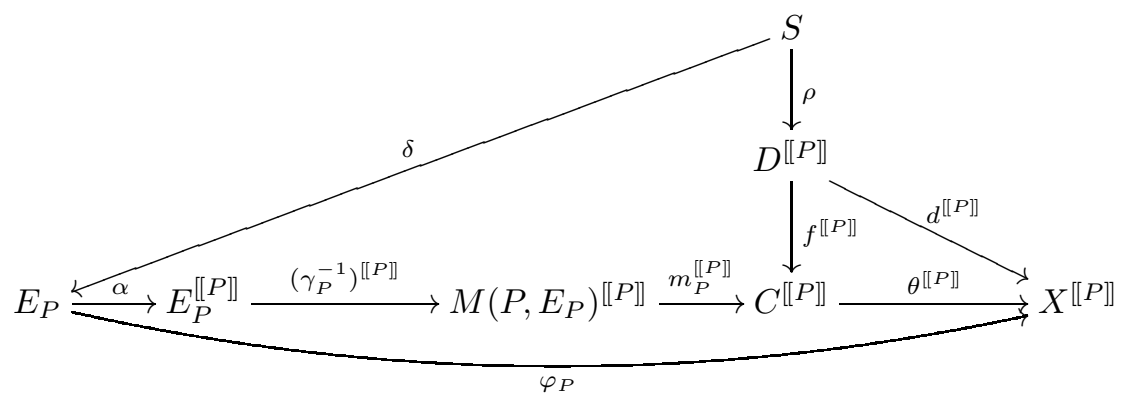

The map $\alpha$ is the canonical projection from $E_{P}=E_{P}{ }^{P}$ to $E_{P}^{[[P]}$. The lower shape commutes because $\varphi_{P}=\varphi_{P}^{\llbracket[P \rrbracket} \alpha$ and $\varphi_{P}^{\llbracket P \rrbracket]} \gamma_{P}^{\llbracket P \rrbracket]}=\theta^{\llbracket P \rrbracket} m_{P}^{\llbracket[P]}$. Note that $\delta$ is surjective because $\varphi_{P} \delta=d^{[P \rrbracket]} \rho$ is a surjective map from the projective $R\left[\mathrm{~N}_{G}(P) / P\right]$-module $S$ to $X^{[[P]]}$ and $\varphi_{P}$ is a projective cover of $X^{\llbracket P]]}$. This yields a contradiction, because the path from $S$ to $C^{[[P \rrbracket}$ going via $\delta$ is surjective, being a composition of surjective maps, while $f^{\llbracket P \rrbracket} \rho$ is not surjective.

The proof of Theorem 6.13 does not suppose the existence of covers and thus provides a proof of the existence of permutation covers independent of Theorem 6.2

We now use the explicit description of the permutation cover to obtain a characterization of $R$ permutation modules. Here and in future, if $U$ is an $R G$-module then $\bar{U}$ denotes the module $U / \pi U$, which may be treated as an $R G$-module or as an $(R / \pi) G$-module, depending on context.

Proposition 6.14. Let $R$ be a complete discrete valuation ring whose residue class field $k=R / \pi$ has characteristic $p$. If $X$ is a coflasque pseudocompact $R G$-lattice and $\bar{X}=X / \pi X$ is a k-permutation $k G$-module, then $X$ itself is an $R$-permutation $R G$-module.

Proof. One may check using the explicit construction of the permutation cover $\theta_{X}: P_{X} \rightarrow X$ that its reduction $\overline{\theta_{X}}: \overline{P_{X}} \rightarrow \bar{X}$ modulo $\pi$ is the permutation cover of $\bar{X}$ using the following facts (cf. [7]): 
- For any finite group $H$, reduction modulo $\pi$ induces a natural bijection between projective $R H$ modules and projective $k H$-modules;

- Since $\pi X$ (being isomorphic to $X$ ) is coflasque, applying $(-)^{P}$ to the short exact sequence $0 \rightarrow$ $\pi X \rightarrow X \rightarrow \bar{X} \rightarrow 0$ yields the isomorphism

$$
\bar{X}^{P} \cong X^{P} /(\pi X)^{P}=X^{P} / \pi X^{P}=\overline{X^{P}}
$$

Hence $X^{[[P]]}=\bar{X}^{[[P]}$.

- Reduction modulo $\pi$ induces a natural bijection between Green correspondents of indecomposable $R$-permutation modules and those of the corresponding indecomposable $k$-permutation modules.

But $\overline{\theta_{X}}$ is an isomorphism by hypothesis. So the kernel of $\theta_{X}$ is a pure submodule of $P_{X}$ contained in $\pi P_{X}$, and hence is 0 . That is, $P_{X} \cong X$ and $X$ is a permutation module.

\section{A theorem of Cliff and Weiss}

From now on, $R$ will be a complete discrete valuation ring whose field of fractions has characteristic 0 and whose residue class field $k=R / \pi$ has prime characteristic $p$. Given an $R G$-lattice $L$ we want to find a small power $\pi^{e}$ such that when $L / \pi^{e} L$ is a direct summand of a permutation module over $R / \pi^{e}$, then $L$ is coflasque. We adapt the argument in [11] to see that if some given $e$ has this property for every lattice for a cyclic group of order $p$, then this same $e$ will work for the whole group $G$. Indeed, we may restrict to the case where $G$ is a $p$-group because $L \mid L \downarrow_{P} \uparrow^{G}$ for any Sylow $p$-subgroup of $G$ so that, by the Eckmann-Shapiro Lemma and Mackey decomposition, we need only check that $L_{\downarrow_{P}}$ is coflasque. Suppose that $G$ is a $p$-group and proceed by induction on its order, the case of order $p$ being by hypothesis. Let $Q$ be a central subgroup of order $p$ in $G$. Since $H^{1}(Q, L)=0$ the sequence

$$
0 \rightarrow L^{Q} \stackrel{\pi^{e}}{\rightarrow} L^{Q} \rightarrow\left(L / \pi^{e} L\right)^{Q} \rightarrow 0
$$

is exact, so that $L^{Q} / \pi^{e} L^{Q} \cong\left(L / \pi^{e} L\right)^{Q}$ is a permutation $\left(R / \pi^{e}\right)[L / Q]$-lattice. By induction, $L^{Q}$ is coflasque as a module for $G / Q$. Now, the inflation-restriction exact sequence (see [35, 6.8.3])

$$
0 \rightarrow H^{1}\left(G / Q, L^{Q}\right) \rightarrow H^{1}(G, L) \rightarrow H^{1}(Q, L)^{G / Q}
$$

shows that $H^{1}(G, L)=0$, as required.

Lemma 7.1. Let $G$ be a finite group and let $L$ be an $R G$-lattice in $R G$-Mod ${ }^{\mathcal{C}}$. Suppose that $F$ is a free $k G$-submodule of $L / \pi L$. Then there is a free $R G$-lattice $\widetilde{F} \leqslant L$ that is a summand of $L$ and such that $\widetilde{F} / \pi \widetilde{F} \cong(\widetilde{F}+\pi L) / \pi L=F$.

Proof. This is well known. Let $\widehat{F} \rightarrow F$ be the projective cover of $F$ as an $R G$-module. The composition $\widehat{F} \rightarrow F \rightarrow L / \pi L$ lifts to $\gamma: \widehat{F} \rightarrow L$ since $\widehat{F}$ is projective. Let $\widetilde{F}=\gamma(\widehat{F})$. Then $\widetilde{F}$ is pure over $R$ in $L$ and so is an $R$-summand of $L$. The induction and coinduction functors being isomorphic for finite groups, and $\gamma$ being injective since it is injective modulo $\pi$, mean that $\widetilde{F}$ is injective relative to the the trivial subgroup of $G$ (see the discussion after Lemma 6.11). Thus, being an $R G$-submodule and an $R$-summand of $L$, it is an $R G$-summand.

Remark: Let $G$ be a $p$-group. Replacing $F$ with a permutation module $V_{H} \uparrow G \leqslant L / \pi L$, let $\widehat{V}_{H}$ be the projective cover of $V_{H}$ as an $R$-module, treated as a trivial $R H$-module. Then $\widehat{V}_{H}$ lifts to $\widetilde{V}_{H} \leqslant L$ if

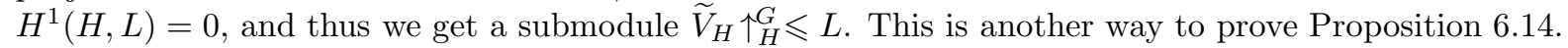

Let $C$ be a group of order $p$ and let $L$ be an $R C$-lattice such that $L / \pi^{e} L$ is a permutation $\left(R / \pi^{e}\right) C$ lattice. The module $L / \pi^{e} L$ decomposes as the sum of a free $\left(R / \pi^{e}\right) C$-lattice and a trivial $\left(R / \pi^{e}\right) C$-lattice. By Lemma 7.1 the free part lifts to a summand of $L$, whose complement we denote by $M$. Note that $M / \pi^{e} M$ is trivial. 
Observe that a short exact sequence of lattices $J \rightarrow M \rightarrow X$ is necessarily split over $R$, so that the reduction $J / \pi^{e} J \rightarrow M / \pi^{e} M \rightarrow X / \pi^{e} X$ is exact, hence if $M / \pi^{e} M$ is trivial then so are $J / \pi^{e} J$ and $X / \pi^{e} X$. The $R C$-lattice $M$ is an inverse limit of lattices of finite rank by Lemma 2.1 and these quotients are necessarily trivial modulo $\pi^{e}$ by the above observation. A lattice of finite rank is an iterated extension of irreducible lattices [15, Cor. 23.15 and $\S 25]$, where by irreducible lattice we mean a lattice $S$ such that $K \otimes_{R} S$ is irreducible as a $K C$-module, $(K$ the field of fractions of $R$ ). Thus if we express each finite rank lattice as an iterated extension of irreducible lattices, then each of these must also be trivial modulo $\pi^{e}$.

The irreducible $K C$-modules are either trivial or of the form $K(\omega)$ for $\omega$ a primitive $p$ th root of unity, where a given generator $c$ of $C$ acts by multiplication by $\omega^{r}$ with $r$ not divisible by $p$. A lattice in such a module corresponds to a fractional ideal of $K(\omega)$ and such ideals are principal. Thus, such a lattice is isomorphic to $R[\omega]$.

We have the following two cases:

- $\omega \notin K$ : Then the extension $K(\omega) / K$ is ramified and so $\omega-1 \notin \pi R[\omega]$. In this case we can take $e=1$ so that the irreducible lattices are trivial. But extensions and inverse limits of trivial lattices are trivial, so that our infinite lattice is also trivial.

- $\omega \in K$, hence $\omega \in R$ : Then $\omega-1 \notin\langle(\omega-1) \pi\rangle$, so the action of $C$ on $R[\omega]$ is not trivial modulo $(\omega-1) \pi$. If we choose $e$ so that $\left\langle\pi^{e}\right\rangle=\langle(\omega-1) \pi\rangle$ then the infinite lattice must have been trivial.

Putting these observations together we obtain a form of a result of [1]:

Theorem 7.2. Let $G$ be a finite group and let $L$ be a lattice in $R G$-Mod ${ }^{\mathcal{C}}$.

1. If $R$ does not contain a primitive pth root of unity then $L$ is an $R$-permutation lattice over $R$ if, and only if, $L / \pi L$ is a $k$-permutation module.

2. If $R$ contains a primitive pth root of unity $\omega$ then $L$ is an $R$-permutation lattice over $R$ if, and only if, $L /(\omega-1) \pi L$ is an $R /(\omega-1) \pi$-permutation lattice.

Observe that, by considering lattices of rank 1, we already see that the power of $\pi$ in part 2 is the best possible.

\section{Weiss' Theorem}

Our goal for this section is to prove Theorem 1.2. A special case of this result was proved in [23] with $R=\mathbb{Z}_{p}$. The missing link needed to obtain the result in generality is Theorem 8.6. a generalization of [36. Thm 3] for infinitely generated lattices. Throughout this section, $G$ is a finite $p$-group and $R$ is a complete discrete valuation ring whose residue class field has characteristic $p$ and whose field of fractions has characteristic 0 . Denote by $\pi$ a generator of the maximal ideal of $R$ and let $k=R / \pi$.

If $U$ is an $R G$-module, recall that by $\bar{U}$ we denote the $k G$-module $U / \pi U$. When $R$ contains a primitive $p$ th root of unity $\omega$, let $\widetilde{R}=R /(\omega-1)$ and denote by $\widetilde{U}$ the $\widetilde{R} G$-module $U /(\omega-1) U$. For $S$ any quotient ring of $R$ (including $R$ ), a pseudocompact $S G$-lattice is said to be diagonal if it is isomorphic to a direct product of indecomposable lattices of rank 1 over $S$ (diagonal is thus a special case of monomial).

Lemma 8.1. Suppose that $R$ contains a primitive pth root of unity $\omega$. If a short exact sequence of pseudocompact $\widetilde{R} G$-modules $0 \rightarrow X^{\prime} \stackrel{s}{\rightarrow} X \stackrel{h}{\rightarrow} X^{\prime \prime} \rightarrow 0$ splits as $\widetilde{R}$-modules and if the middle term is diagonal, then the sequence splits as $\widetilde{R} G$-modules.

Proof. We mimic the proof of [38, Lem. 52.2]. Write $X=\prod_{I} X_{i}$ with each $X_{i}$ an $\widetilde{R} G$-sublattice of $X$ of $\widetilde{R}$-rank 1 . The sequence remains split over $\bar{R}$ and the $\overline{X_{i}}$ are indecomposable $\bar{R}$-modules, so by Proposition 3.5 applied to these $\bar{R}$-modules there is a subset $J$ of $I$ such that $\bar{X}=\bar{s}\left(\overline{X^{\prime}}\right) \oplus \prod_{J} \overline{X_{i}}$, and this remains true over $\bar{R} G$. Thus the $\bar{R} G$-module homomorphism $\bar{h}^{\prime}=\left.\bar{h}\right|_{\prod_{J}} \overline{X_{i}}$ is an isomorphism, where $\bar{h}$ is the map induced by $h$. By Nakayama's Lemma, $\left.h\right|_{\prod_{J} X_{i}}=h^{\prime}: \prod_{J} X_{i} \rightarrow X^{\prime \prime}$ is an isomorphism. Now the composition of $h^{\prime-1}$ with the inclusion $\prod_{J} X_{i} \rightarrow X$ is a splitting of $h$. 
In the above proof we work modulo $\pi$ because there need not be finitely many isomorphism classes of rank $1 \widetilde{R} G$-lattice, so that Proposition 3.5 need not apply directly to $X$.

Proposition 8.2. Suppose that $R$ contains a primitive pth root of unity $\omega$. Let $U$ be a pseudocompact $R G$-lattice and $V$ a diagonal pseudocompact $R G$-lattice. If $\widetilde{U} \cong \widetilde{V}$, then $U$ is diagonal.

Proof. Write $U=\lim _{i} U / X_{i}$ with each $U / X_{i}$ an $R G$-lattice of finite rank over $R$, as we may by Lemma2.1 As there are only finitely many isomorphism classes of indecomposable diagonal $R G$-lattices, by Theorem 3.8 applied with $M$ the direct sum of a representative of each isomorphism class of indecomposable diagonal $R G$-lattice, it suffices to check that each $U / X_{i}$ is diagonal. The sequence $0 \rightarrow X_{i} \rightarrow U \rightarrow$ $U / X_{i} \rightarrow 0$ is split over $R$ because $U / X_{i}$ is a lattice, so applying $\widetilde{(-)}$ it follows that $\widetilde{U / X_{i}} \cong \widetilde{U} / \widetilde{X_{i}}$. Denoting by $\rho: \widetilde{U} \rightarrow \widetilde{V}$ the given isomorphism, we have

$$
\widetilde{U / X_{i}} \cong \widetilde{U} / \widetilde{X_{i}} \cong \widetilde{V} / \rho\left(\widetilde{X_{i}}\right) .
$$

By Lemma $8.1, \widetilde{V} / \rho\left(\widetilde{X_{i}}\right)$ is a direct summand of $\widetilde{V}$, hence diagonal by the Krull-Schmidt property. It follows that the finitely generated $R G$-lattice $U / X_{i}$ is diagonal modulo $\omega-1$, and hence is diagonal by the finitely generated version of this result [38, Thm 50.2] (in fact, we only require Case B in the proof). Note that the cited proof is for compact discrete valuation rings, but the same proof holds for complete discrete valuation rings because we still have Nakayama's Lemma.

Lemma 8.3. Suppose that $f: L \rightarrow M$ is a (continuous) homomorphism of pseudocompact $R$-lattices such that $\bar{f}: \bar{L} \rightarrow \bar{M}$ is injective. Then $f$ is a split injection in $R$-Mod ${ }^{\mathcal{C}}$.

Proof. If $f$ is not injective, then there exists $x \neq 0$ in the kernel. Write $x=\pi^{n} y$ for some $y \notin \pi L$. Now

$$
0=f(x)=f\left(\pi^{n} y\right)=\pi^{n} f(y),
$$

so that $f(y)=0$. Hence there exists $y \notin \pi L$ in the kernel of $f$. But now

$$
\bar{f}(\bar{y})=\overline{f(y)}=0,
$$

contradicting the injectivity of $\bar{f}$.

We claim that $f(L)$ is pure in $M$. If not, then there is $y \in L \backslash \pi L$ such that $f(y)=\pi m$ for some $m \in M$. Now $\bar{y} \neq 0$, while

$$
\bar{f}(\bar{y})=\overline{f(y)}=0,
$$

contradicting the injectivity of $\bar{f}$. Thus $M / f(L)$ is torsion-free, hence free, and so $f$ splits.

We next generalize [38, Prop. 53.2] to infinite rank lattices:

Lemma 8.4. Suppose we have a short exact sequence of RG-lattices

$$
0 \rightarrow L \rightarrow T \stackrel{\gamma}{\rightarrow} M \rightarrow 0
$$

with $L, M$ monomial and such that the induced sequence

$$
0 \rightarrow \widetilde{L} \rightarrow \widetilde{T} \rightarrow \widetilde{M} \rightarrow 0
$$

is split exact. Then the sequence splits.

Proof. We first show that $\gamma$ is monomial supersurjective. Writing $\left(\varphi \uparrow_{H}^{G},-\right)$ instead of $\operatorname{Hom}_{R G}\left(\varphi \uparrow_{H}^{G},-\right)$ and applying it to the given sequence, we obtain

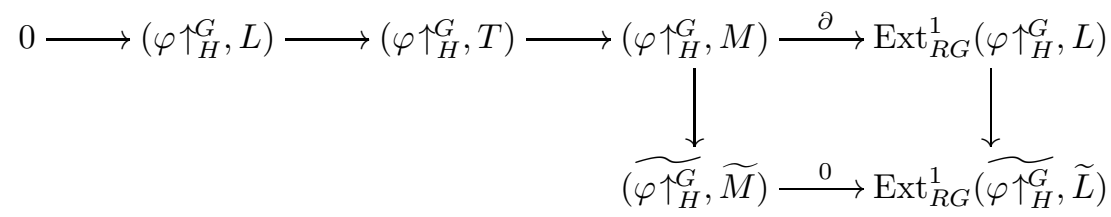


Write $L=\prod L_{i}$ as a product of indecomposable monomial modules. We have

$$
\operatorname{Ext}_{R G}^{1}\left(\varphi \uparrow_{H}^{G}, L\right)=\prod \operatorname{Ext}_{R G}^{1}\left(\varphi \uparrow_{H}^{G}, L_{i}\right)
$$

By [38, Prop. 53.2], on each factor of this product the vertical right map is injective, and hence the vertical right map is itself injective. But this forces $\partial$ to be 0 , showing that $\left(\varphi \uparrow_{H}^{G}, T\right) \rightarrow\left(\varphi \uparrow_{H}^{G}, M\right)$ is surjective, so that $\gamma$ is monomial supersurjective.

By Lemma 6.5 the monomial supersurjectivity of $\gamma$ implies that $(M, \gamma):(M, T) \rightarrow(M, M)$ is surjective. An element of the preimage of $\operatorname{id}_{M}$ is the required splitting of $\gamma$.

We need the following standard lemma (see after Lemma 6.11 for definitions).

Lemma 8.5. Let $U$ be a finite direct sum of modules $U_{i}$ each projective relative to a proper subgroup of $G$. Suppose that the epimorphism $\gamma: Y \rightarrow U$ splits over every proper subgroup of $G$. Then $\gamma$ splits.

Proof. Suppose that each $U_{i}$ is relatively $H_{i}$-projective for $H_{i}<G$. Then $\operatorname{id}_{U_{i}}=\operatorname{Tr}_{H_{i}}^{G}\left(\alpha_{i}\right)$ for some $\alpha_{i} \in \operatorname{End}_{R H_{i}}\left(U_{i}\right)$. Hence $\operatorname{id}_{U}=\sum_{i} \operatorname{Tr}_{H_{i}}^{G}\left(\alpha_{i}\right)$. For each $i$, let $s_{i}$ be a splitting of $\gamma$ as an $H_{i}$-module homomorphism. Using [3, Lem. 3.6.3] we obtain

$$
\gamma\left(\sum_{i} \operatorname{Tr}_{H_{i}}^{G}\left(s_{i} \alpha_{i}\right)\right)=\sum_{i} \operatorname{Tr}_{H_{i}}^{G}\left(\gamma s_{i} \alpha_{i}\right)=\sum_{i} \operatorname{Tr}_{H_{i}}^{G}\left(\alpha_{i}\right)=\mathrm{id}_{U} .
$$

The next theorem is a generalization of [38, Thm 50.2] to pseudocompact modules.

Theorem 8.6. Let $R$ be a complete discrete valuation ring containing a primitive pth root of unity $\omega$. If $U$ is a pseudocompact lattice such that $\widetilde{U}$ is monomial, then $U$ itself is monomial.

Proof. We work by induction on the order of $G$. If $|G|=1$ the result is obvious. We suppose that the result holds for any proper subgroup of $G$.

Applying the functor $\widetilde{(-)}$ to the monomial cover $c: P \rightarrow U$ yields the commutative square

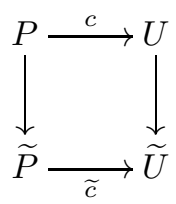

For any proper subgroup $H$ of $G$ the homomorphism $c \downarrow_{H}$ is a surjection from the monomial module $P \downarrow_{H}$ to the module $U \downarrow_{H}$, the latter monomial by induction. Using Frobenius reciprocity, we see that $c \downarrow_{H}$ is a monomial precover. Hence, since $U \downarrow_{H}$ is monomial, the homomorphism $c \downarrow_{H}$ is a split surjection for every proper subgroup $H$ of $G$. Thus $\widetilde{c}$ also splits over every proper subgroup of $G$.

The module $\widetilde{U}$ is monomial by hypothesis, so write it as $T \oplus Y$, where $T$ is diagonal and $Y$ is projective relative to proper subgroups of $G$. Consider the composition

$$
\widetilde{P} \stackrel{\widetilde{c}}{\rightarrow} T \oplus Y \stackrel{\pi_{Y}}{\longrightarrow} Y .
$$

This homomorphism splits over every proper subgroup of $G$ (because $\widetilde{c}$ does) and hence, since $Y$ is projective relative to proper subgroups, it splits over $G$ (to see this, apply Lemma 8.5 to $Y$ written as a finite sum of modules each projective relative to a fixed maximal subgroup of $G$, for example). Let $\beta: Y \rightarrow \widetilde{P}$ be an $\widetilde{R} G$-module homomorphism such that $\pi_{Y} \widetilde{c} \beta=\mathrm{id}_{Y}$. Define the following submodules of $\widetilde{P}:$

$$
\begin{gathered}
Y^{\prime}=\beta(Y), \\
T^{\prime}=\widetilde{c}^{-1}(T) .
\end{gathered}
$$


Routine checks show that $\widetilde{P}=Y^{\prime} \oplus T^{\prime}$. Observe that $\left.\widetilde{c}\right|_{Y^{\prime}}: Y^{\prime} \rightarrow Y$ is an isomorphism.

Write $P=\prod_{i \in I} P_{i}$ with the $P_{i}$ indecomposable monomial lattices. Hence $\widetilde{P}=\prod_{I} \widetilde{P}_{i}$. This module has the exchange property (Proposition [3.5 can be applied because there are finitely many isomorphism classes of indecomposable monomial $R G$-lattice, hence finitely many $\widetilde{P}_{i}$ up to isomorphism), so we can write

$$
\widetilde{P}=\prod_{i \in J} \widetilde{P}_{i} \oplus T^{\prime}
$$

for some subset $J \subseteq I$.

Let $W=\prod_{J} P_{i}$ be the corresponding summand of $P$. It follows that $\widetilde{P}=\widetilde{W} \oplus T^{\prime}$, and hence that

$$
\widetilde{W} \cong \widetilde{P} / T^{\prime} \cong Y^{\prime}
$$

The reader may check that $\widetilde{c} \widetilde{W}_{\widetilde{W}}$ is injective. We claim that $\widetilde{U}=\widetilde{c}(\widetilde{W}) \oplus T$ : That $\widetilde{U}=\widetilde{c}(\widetilde{W})+T$ is obvious, so consider $u \in \widetilde{c}(\widetilde{W}) \cap T$, and write $u=\widetilde{c}(w)$ for $w \in \widetilde{W}$. Then

$$
w \in \widetilde{W} \cap \widetilde{c}^{-1}(T)=\widetilde{W} \cap T^{\prime}=0,
$$

so that $u=\widetilde{c}(0)=0$.

We have the following diagram:

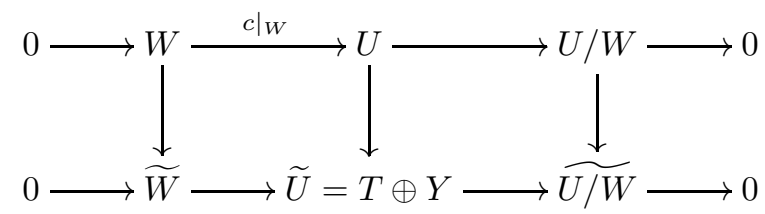

The second row is exact because $\widetilde{(-)}$ is right exact and $\widetilde{c} \widetilde{W}_{\overparen{W}}$ is injective. It follows from the claim above that $\widetilde{U / W} \cong T$. Thus, by Proposition 8.2, $U / W$ is monomial. Meanwhile, $W$ is monomial by construction.

Finally, the lower sequence splits $\left(\widetilde{c} \widetilde{W}_{W}\right.$ is a split injection) so by Lemma 8.4 $U \cong W \oplus U / W$ is monomial, as required.

We prove Theorem 1.2. We work by induction on the order of $G$. If $N=1$ the result is immediate so suppose that $N \neq 1$. There exists a central normal subgroup $M$ of $G$ of order $p$ contained in $N$. Consider the $G / M$-module $U^{M}$. We have $\left(U^{M}\right)^{N}=U^{N}$ is a permutation $G / N=(G / M) /(N / M)$-module, and $U^{M} \downarrow_{N / M} \cong\left(U \downarrow_{N}\right)^{M}$ is a free $N / M$-module. Hence by induction we have that $U^{M}$ is a permutation $G / M$-lattice. But we also have that $U \downarrow_{M}=U \downarrow_{N} \downarrow_{M}$ is free, and hence we need only show that the theorem holds when $N$ is central of order $p$. We assume this from now on. Denote by $\Sigma_{N}$ the element $\sum_{n \in N} n \in R N$.

Lemma 8.7. Let $N$ be a central subgroup of $G$ of order $p$ and let $U$ be a pseudocompact $R G$-lattice such that $U^{N}$ is a permutation module and $U \downarrow_{N}$ is free. Let $L$ be a pseudocompact permutation RG-lattice and let $f: L \rightarrow U / \Sigma_{N} U$ be a surjection having kernel $\Sigma_{N} L$. Then $L \cong U$.

Proof. Note that $R N / R N^{N}$ is torsion-free (as an $R$-module), so $U / U^{N}$ is torsion-free since $U \downarrow_{N}$ is free. Note also that since $U$ is free over $N$, we have $\Sigma_{N} U=U^{N}$. Write $L=\prod_{i} R\left[G / H_{i}\right]$ (via Proposition 2.8). If for some $i$ we had $N \leqslant H_{i}$, then by the Mackey decomposition, $L \downarrow_{N}$ would have a trivial summand $R$. But then $R / \Sigma_{N} R=R / p R$ would be isomorphic to a submodule of $U / \Sigma_{N} U$-impossible, since $U / \Sigma_{N} U$ is torsion-free. Hence $L \downarrow_{N}$ is free.

Because $\Sigma_{N} U=U^{N}$ is a permutation module by hypothesis, we have $H^{1}\left(H, \Sigma_{N} U\right)=0$ for all $H$ by Lemma 2.9. Thus the homomorphism $U \rightarrow U / \Sigma_{N} U$ is supersurjective and hence, by Lemma 6.4, $f$ lifts to a homomorphism $\widehat{f}: L \rightarrow U$. 
Consider the triangle

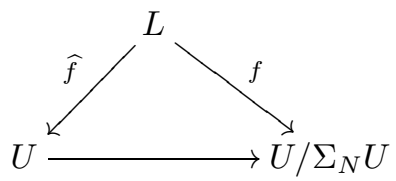

Considering this triangle modulo $\Sigma_{N}$ shows that $\widehat{f}$ is an isomorphism modulo $\Sigma_{N}$. But $R N$ is local, hence $\widehat{f}$ is surjective by Nakayama's Lemma and injective by Lemma 8.3 ,

By the discussion before Lemma 8.7, Theorem 1.2 follows from the following special case:

Theorem 8.8. Let $R$ be a discrete valuation ring whose field of fractions has characteristic 0 and whose residue field has characteristic $p>0$. Let $G$ be a finite $p$-group and let $U$ be a pseudocompact $R G$-lattice. Suppose $G$ has a central subgroup $N$ of order $p$ such that

- $U \downarrow_{N}$ is free,

- $U^{N}$ is a permutation module.

Then $U$ itself is a permutation module.

Proof. We prove this first in the special case that $p$ is prime in $R$. Adjoin a primitive $p$ th root of unity $\omega$ to $R$ to obtain the ring $R[\omega]=R[x] /\left(1+x+\ldots+x^{p-1}\right)$. The kernel of the surjective ring homomorphism $R[\omega] \rightarrow R / p$ sending $\omega$ to 1 is generated by $\omega-1$, so that $p$ prime in $R$ implies that $\omega-1$ is prime in $R[\omega]$. If $p=2$ then $R[\omega]=R$, but this does not affect the argument. Fix an isomorphism of groups $\psi: N \rightarrow\langle\omega\rangle$. If $p$ is odd, define the structure of $R[\omega] G$-lattice on the module $U / \Sigma_{N} U$ via $\psi$ by defining the action of $\omega$ as

$$
\omega \cdot\left(u+\Sigma_{N} U\right):=\psi^{-1}(\omega) u+\Sigma_{N} U,
$$

(if $p$ is 2 there is nothing to define, but in this case note that the formula agrees with the action of $\omega=-1$ on $\left.U / \Sigma_{N} U\right)$. This structure is natural in the sense that having fixed $\psi$, we can consider $U \mapsto U / \Sigma_{N} U$ as a functor $R G$-Mod $\rightarrow R[\omega] G$-Mod. Multiplication by $\Sigma_{N}$ yields an isomorphism

$$
\left(U / \Sigma_{N} U\right) /(\omega-1)\left(U / \Sigma_{N} U\right) \cong U^{N} / p U^{N} .
$$

This is a permutation module (because $U^{N}$ is), and hence by Theorem 8.6, $U / \Sigma_{N} U$ is a monomial $R[\omega] G$-lattice. So write

$$
U / \Sigma_{N} U=\prod_{i \in I} V_{i} \uparrow_{H_{i}}^{G},
$$

where $V_{i}$ is an $R[\omega] H_{i}$ lattice of rank 1 . As $R[\omega]$ contains no primitive $p^{2}$ root of unity, the action of each $H_{i}$ on the corresponding $V_{i}$ is given by a group homomorphism $\varphi_{i}$ from $H_{i}$ to $\langle\omega\rangle$. Denote by $K_{i}$ the kernel of $\varphi_{i}$. The $R N$-module $R N / \Sigma_{N} R N$ has no non-zero $N$-fixed points and hence, since $U$ is $N$-free, $U / \Sigma_{N} U$ has no non-zero $N$-fixed points. Calculating the $N$-fixed points of $V_{i} \uparrow_{H_{i}}^{G}$ differently, we obtain isomorphisms of $R$-modules

$$
\begin{aligned}
0=\left(V_{i} \uparrow_{H_{i}}^{G} \downarrow_{N}\right)^{N} & \cong \bigoplus_{g \in G / H_{i} N} \operatorname{Hom}_{N}\left(R,{ }^{g} V_{i} \downarrow_{g H_{i} \cap N} \uparrow^{N}\right) \\
& \cong \bigoplus_{g \in G / H_{i} N} \operatorname{Hom}_{g_{H_{i}} \cap N}\left(R,{ }^{g} V_{i} \downarrow_{{ } H_{i} \cap N}\right) .
\end{aligned}
$$

It follows that $H_{i} \cap N$ is not contained in $K_{i}$ and hence that $N$ is a subgroup of $H_{i}$ not intersecting $K_{i}$. In particular, $\varphi_{i}$ is not trivial.

A surjective $R H_{i}$-module homomorphism $R\left[H_{i} / K_{i}\right] \rightarrow V_{i} /(\omega-1) V_{i} \cong R / p$ lifts by projectivity over $H_{i} / K_{i}$ to a surjective $R H_{i}$-module homomorphism from $R\left[H_{i} / K_{i}\right]$ to $V_{i}$. Inducing to $G$, we obtain a 
surjective $R G$-module homomorphism $R\left[G / K_{i}\right] \rightarrow V_{i} \uparrow_{H_{i}}^{G}$ whose kernel contains (hence is equal to by comparison of ranks) $\Sigma_{N} R\left[G / K_{i}\right]$, so we have an isomorphism of $R G$-modules

$$
R\left[G / K_{i}\right] / \Sigma_{N} R\left[G / K_{i}\right] \cong V_{i} \uparrow_{H_{i}}^{G} .
$$

Define the permutation lattice $L=\prod_{i \in I} R\left[G / K_{i}\right]$ together with the obvious homomorphism $L \rightarrow$ $U / \Sigma_{N} U$, whose kernel is $\Sigma_{N} L$. Now by Lemma 8.7. $U \cong L$ is a permutation lattice. This completes the special case.

For the general case, let $S$ be a coefficient ring for $R$, in the sense of Cohen's Structure Theorem [13. Thus $S$ is a complete discrete valuation ring contained in $R$ with the following properties:

- The inclusion $S \rightarrow R$ realizes $R$ as a free pseudocompact $S$-module of finite rank over $S$ (cf. [32, Ch. $1 \S 6])$.

- The number $p$ generates the maximal ideal of $S$.

Denote by $U^{\prime}$ the module $U$ considered as an $S G$-module. Then $U^{\prime}$ satisfies the hypotheses of the theorem, so that $U^{\prime}$ is a permutation $S G$-module by the special case above. As $U^{\prime}$ is free over $N$, it is a product of modules $S[G / H]$ with $H \cap N=1$. Since id : $U^{\prime} \rightarrow U^{\prime}$ is the permutation cover of $U^{\prime}$, we have that $R \widehat{\otimes}_{S} U^{\prime} \rightarrow U$ given by $r \widehat{\otimes} u \mapsto r u$ is supersurjective, so is a permutation precover of $U$ by Lemma 6.4. It follows that the permutation cover of $U$ (a direct summand of $R \widehat{\otimes}_{S} U^{\prime}$ ) is free on restriction to $N$. Denote by

$$
0 \rightarrow K \rightarrow C \stackrel{f}{\rightarrow} U \rightarrow 0
$$

the permutation cover of $U$. We will show that $K=0$. By freeness of $U$ over $N$ the sequence is split over $N$ and hence

$$
0 \rightarrow K_{N} \rightarrow C_{N} \stackrel{f_{N}}{\longrightarrow} U_{N} \rightarrow 0
$$

is exact. Note that $U_{N}$ is a permutation module and $f_{N}$ is supersurjective, both these claims following from the natural isomorphisms $C_{N} \rightarrow C^{N}$ and $U_{N} \rightarrow U^{N}$ given by multiplication by $\Sigma_{N}$, again by freeness of $C$ and $U$ over $N$. Thus $f_{N}$ is a permutation precover of the permutation module $U_{N}$, so it splits and hence $K_{N}$ is a direct summand of $C_{N}$. Write $C=\prod_{I} V_{i}$, a product of indecomposable modules. Then each $\left(V_{i}\right)_{N}$ is indecomposable, and $C_{N}=\prod_{I}\left(V_{i}\right)_{N}$. By the extension property, there is a subset $J$ of $I$ such that $C_{N}=K_{N} \oplus \prod_{J}\left(V_{i}\right)_{N}$. By Nakayama's Lemma, $C=K+\prod_{J} V_{i}$. The restriction $f: \prod_{J} V_{i} \rightarrow U$ is thus also a precover of $U$, so that $I=J$ since $f$ is a cover. It follows that $K_{N}=0$, hence $K=0$ as required.

\section{References}

[1] F.W. Anderson, K.R. Fuller, Rings and Categories of Modules, Springer-Verlag 1992.

[2] L. Bary-Soroker, M. Jarden and D. Neftin, The Sylow subgroups of the absolute Galois group Gal( $(\mathbb{Q})$. Adv. Math. 284 (2015), 186-212.

[3] D.J. Benson, Representations and Cohomology I: Basic representation theory of finite groups and associative algebras, Cambridge University Press 1995.

[4] G.M. Bergman, Every module is an inverse limit of injectives, Proceedings of the American Mathematical Society, 141, (2012), 1177-1183.

[5] M. Boggi and G. Corob Cook, Continuous cohomology and homology of profinite groups, Documenta Mathematica, 21, (2016), 1269-1312.

[6] R. Boltje and R. Kessar and M. Linckelmann, On Picard groups of blocks of finite groups, (2019), accepted in Journal of Algebra, https://doi.org/10.1016/j.jalgebra.2019.02.045. 
[7] M. Broué, On Scott modules and p-permutation modules: an approach through the Brauer morphism, Proceedings of the American Mathematical Society 93, (1985), 401-408.

[8] K.S. Brown, Cohomology of Groups, Springer 1982.

[9] A. Brumer, Pseudocompact algebras, profinite groups and class formations, Journal of Algebra, 4, (1966), 442-470.

[10] S.U. Chase, Direct products of modules, Transactions of the American Mathematical Society, 97, (1960), 457-473.

[11] G. Cliff and A. Weiss, Summands of permutation lattices for finite groups, Proceedings of the American Mathematical Society, 110, (1990), 17-20.

[12] D.E. Cohen, Groups with free subgroups of finite index, in: Conference on Group Theory, University of Wisconsin-Parkside 1972, Lecture Notes in Mathematics 319, Springer-Verlag (1973), pp. 26-44.

[13] I.S. Cohen, On the structure and ideal theory of complete local rings, Transactions of the American Mathematical Society, 59, (1946), 54-106.

[14] G. Corob Cook, Bieri-Eckmann criteria for profinite groups, Israel Journal of Mathematics, 212, (2015), 857-893.

[15] C.W. Curtis and I. Reiner, Methods of Representation Theory, Vol. 1, John Wiley \& Sons 1981.

[16] F. Eisele, On the geometry of lattices and finiteness of Picard groups, ArXiv eprint 1908.00129, (2019)

[17] E.E. Enochs and O.M.G. Jenda, Relative Homological Algebra, Walter de Gruyter 2000.

[18] P. Gabriel, Des catégories abéliennes, Bulletin de la Société Mathématique de France, 90, (1962), 323-448.

[19] A. Karrass, A. Pietrovski and D. Solitar, Finite and infinite cyclic extensions of free groups, Journal of the Australian Mathematical Society, 16, (1973), 458-466.

[20] T.Y. Lam, A First Course in Noncommutative Rings, Springer 2001.

[21] M. Livesey, On Picard groups of blocks with normal defect groups, ArXiv eprint 1907.12167, (2019)

[22] J.W. MacQuarrie, Modular representations of profinite groups, Journal of Pure and Applied Algebra, 215, (2011), 753-763.

[23] J.W. MacQuarrie and P.A. Zalesskii, Second countable virtually free pro- $p$ groups whose torsion elements have finite centralizer, Selecta Mathematica, 23, (2017), 101-115.

[24] H. Matsumura, Commutative Ring Theory, Cambridge University Press 1989.

[25] O.V. Mel'nikov. Aspherical pro-p-groups. (Russian) Matematicheskii Sbornik 193 (2002), 71-104; translation in Sbornik. Mathematics 193 (2002), 1639-1670.

[26] L. Puig, On the local structure of Morita and Rickard equivalences between Brauer blocks, Birkhäuser Verlag, 1999.

[27] L. Ribes and P.A. Zalesskii, Profinite Groups, Springer 2010.

[28] K. Roggenkamp, Subgroup rigidity of $p$-adic group rings (Weiss arguments revisited), Journal of the London Mathematical Society (2), 46, (1992), 432-448.

[29] M. Samy Modeliar, Certaines contructions liées aux foncteurs de Mackey cohomologiques, thesis, Université Paris 7, 2005. 
[30] M. Samy Modeliar, A characterization of permutation modules, Journal of Algebra, 379, (2008), $4472-4484$.

[31] G.P. Scott, An embedding theorem for groups with a free subgroup of finite index, Bulletin of the London Mathematical Society, 6, (1974), 304-306.

[32] J.P. Serre, Local Fields, Springer 1979.

[33] P. Symonds, Permutation complexes for profinite groups, Commentarii Mathematici Helvetici, 82, (2007), 1-37.

[34] P. Symonds, Structure theorems over polynomial rings, Advances in Mathematics, 208, (2007), 408-421.

[35] C.A. Weibel, An Introduction to Homological Algebra, Cambridge University Press 1994.

[36] A. Weiss, Rigidity of $p$-adic p-torsion, Annals of Mathematics (2) 27, (1988), 317-332.

[37] A. Weiss, Torsion units in integral group rings, Journal für die reine und angewandte Mathematik 415, (1991), 175-188.

[38] A. Weiss, Rigidity of $\pi$-adic $p$-torsion, Appendix in K.S. Sehgal, Units in Integral Group Rings, Chapman and Hall/CRC 1993.

[39] P.A. Zalesskii, Infinitely generated virtually free pro- $p$ groups and $p$-adic representations, Journal of Topology, 12, (2019), 79-93. 